This item was submitted to Loughborough's Research Repository by the author.

Items in Figshare are protected by copyright, with all rights reserved, unless otherwise indicated.

\title{
Effects of swirl on intermittency characteristics in non-premixed flames
}

PLEASE CITE THE PUBLISHED VERSION

http://dx.doi.org/10.1080/00102202.2011.653460

PUBLISHER

(C) Taylor \& Francis

VERSION

AM (Accepted Manuscript)

LICENCE

CC BY-NC-ND 4.0

REPOSITORY RECORD

Ranga-Dinesh, K.K.J., K.W. Jenkins, M.P. Kirkpatrick, and W. Malalasekera. 2019. "Effects of Swirl on Intermittency Characteristics in Non-premixed Flames". figshare. https://hdl.handle.net/2134/10691. 
This item was submitted to Loughborough's Institutional Repository (https://dspace.lboro.ac.uk/) by the author and is made available under the following Creative Commons Licence conditions.

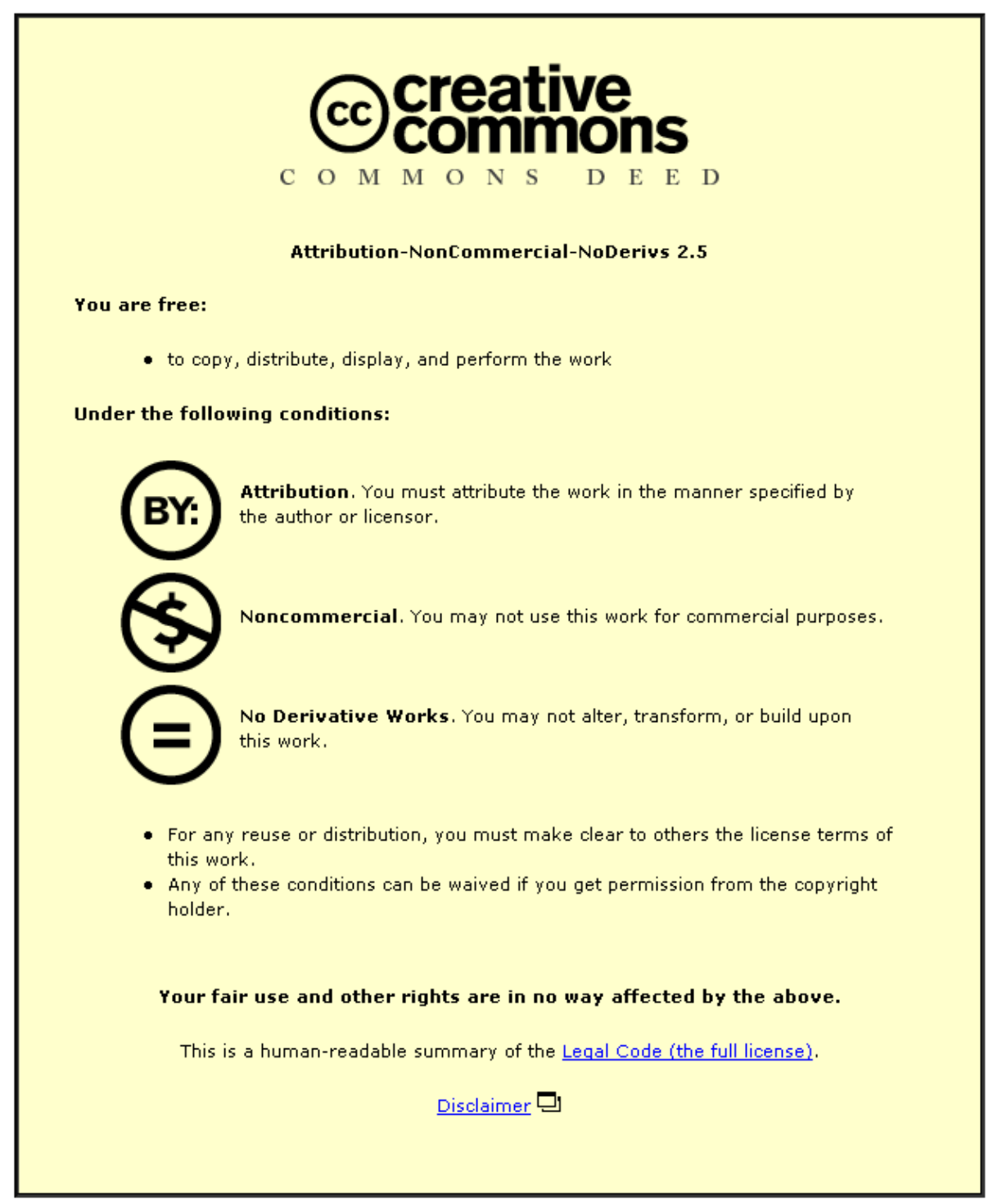

For the full text of this licence, please go to: http://creativecommons.org/licenses/by-nc-nd/2.5/ 


\section{Effects of Swirl on Intermittency Characteristics in Non- Premixed Flames}

K.K.J. Ranga Dinesh ${ }^{1}$, K.W. Jenkins ${ }^{2}$, M.P. Kirkpatrick ${ }^{3}$, W.Malalasekera ${ }^{4}$

1. Engineering Department, Lancaster University, Lancaster, Lancashire, LA1 4YR, UK.

2. School Of Engineering, Cranfield University, Cranfield, Bedford, MK43 0AL, UK.

3. School of Aerospace, Mechanical and Mechatronic Engineering, The University of Sydney, NSW 2006, Australia.

4. Wolfson School of Mechanical and Manufacturing Engineering, Loughborough University, Loughborough, Leicestershire, LE11 3TU, UK.

Corresponding author: K.K.J.Ranga Dinesh

Email address: Ranga.Dinesh@lancaster.ac.uk

Postal Address: Engineering Department, Lancaster University, Lancaster, Lancashire, LA1 4YR, UK.

Telephone number: +44 (0) 1524594578

Revised Manuscript Prepared for the Journal of Combustion Science and Technology $21^{\text {st }}$ December 2011 


\title{
Effects of Swirl on Intermittency Characteristics in Non-
}

\section{Premixed Flames}

\begin{abstract}
:
Swirl effects on velocity, mixture fraction and temperature intermittency have been analysed for turbulent methane flames using LES. The LES solves the filtered governing equations on a structured Cartesian grid using a finite volume method, with turbulence and combustion modelling based on the localised dynamic Smagorinsky and the steady laminar flamelet models respectively. Probability density function (pdf) distributions demonstrate a Gaussian shape closer to the centreline region of the flame and a delta function at the far radial position. However, non-Gaussian pdfs are observed for velocity and mixture fraction on the centreline in a region where centre jet precession occurs. Non-Gaussian behaviour is also observed for the temperature pdfs close to the centreline region of the flame. Due to the occurrence of recirculation zones, the variation from turbulent to non turbulent flow is more rapid for the velocity than the mixture fraction and therefore indicates how rapidly turbulence affects the molecular transport in these regions of the flame.
\end{abstract}

Key Words: Intermittency, Swirl, Non-Premixed Combustion, LES 


\section{Introduction:}

Swirling flames are commonly used in a variety of practical combustion systems, including diesel and gas turbine engines and industrial furnaces (Syred and Beer, 1974, Lucca-Negro and O'Doherty, 2001). Recirculation zones and vortex breakdown (VB) regions are usually found in many turbulent swirling flames and it is well known that swirl stabilised flames are effective in providing a source of well mixed combustion products. These regions also provide storage of heat and chemically active species to sustain combustion (Gupta et al. 1984, Escudier and Keller). Precession motion and precessing vortex core (PVC) structures also occur with certain conditions in swirling flows (Syred, 2006). Investigation of the intermittency in a turbulent swirling flame is particularly interesting due to the different flow structures and various mixing rates that act to form different regimes in a turbulent flame. In particular, unconfined turbulent swirling flames display an intermittent behaviour in some regions such as inside the recirculation zones and close to the outer edge where flow alternates between turbulent and irrotational states (external intermittency) and also due to differences of energy or scalar dissipation rates (internal intermittency). Mathematically an indicative function can be used to identify the external intermittency in such a way that it has a value of one in the turbulent region and zero in the irrotational region. In other words, external intermittency represents the fraction of time during which a point is inside the turbulent field. The dividing interface between turbulent and laminar regions in a turbulent flame is sharp and constantly distorted by turbulent eddies of different sizes, with the turbulent flame propagating into the irrotational region while laminar fluid is entrained into the turbulent region. Therefore a study of intermittency in swirling flames is important and challenging due to the nature of the multi-scale and multi-physics environment. Numerous experimental, theoretical and numerical investigations of turbulent intermittency have been carried out for both reacting and non-reacting applications in fairly simple geometries. The 
first intermittency measurements for a turbulent wake generated by a circular object were undertaken by Townsend (1948) and a similar technique was also used by Corrsin and Kistler (1955) to measure the intermittency in a round jet, and by Klebanoff (1955) in a boundary layer with zero pressure gradient. Wygnanski and Fielder (1969) reported the measurements for a jet using velocity gradients with respect to time with a similar technique applied by Chevray and Tutu (1978) to measure the intermittency of a jet. Bilger et al. (1976) proposed a method using probability density functions (pdf) to overcome some of the difficulties encountered for the calculation of intermittency by the previous investigators. Nakamura et al. (1989) and Schefer and Dibble (2001) adopted Bilger's method to evaluate intermittency for their experiments. Major theoretical work on intermittent flows was initially carried out by Libby (1975), Dopazo (1977), and Chevray and Tutu (1978). Later, Sreenivasan (1985), Jimenez (2000), Gibbson and Doering (2003) and Li and Meneveau (2006) derived various theoretical explanations for turbulent intermittency. Although the majority of turbulence models currently in use were derived for fully developed flows, a few groups have developed and applied Reynolds Averaged Navier-Stokes (RANS) based intermittency models especially for non-reacting turbulent jets. For example, a k-epsilon model based intermittency model was developed by Byggstoyl and Kollomann (1981) and Kollmann and Janicka (1982) who studied intermittency using the transport pdf. Cho and Chung (1992) developed a more economical intermittency model by incorporating an intermittency transport equation into an already existing k-epsilon turbulence model. Few groups extended the Cho and Chung (1992) intermittency model applying it to different applications such as an axisymmetric plume (1997), and a plane plume (2000). Pope (1984) also calculated intermittency using a velocity composition transported probability density function. 
Despite the success of various modelling efforts on turbulence and combustion, Kerstein (2002) outlined many important modelling challenges of turbulence in combustion processes. As explained by Kerstein (2002), there are certain areas in turbulent combustion that are poorly understood. Intermittency is one such topic that involves several unresolved fundamental issues with regard to the representation of large and small scale turbulence effects on turbulent non-premixed and premixed combustion applications. For example, Pedersen et al. (2005) outlined characteristics features of the inhomogeneous interface between turbulent-nonturbulent regions of jets and showed the existence of a finite jump in the tangential velocity at the interface. Furthermore, they have concluded that the engulfment of irrotational fluid is not the dominant process for the entrainment of irrotational fluid in a turbulent jet. Very recently Da Silva (2009) carried out DNS and LES calculations only for temporally evolving turbulent jets and analysed the behaviour of sub-grid scale models near the turbulent-nonturbulent interface. They found strong inhomogeneity of the flow near the jet edge by comparing conditional statistics to demonstrate the differences between various LES sub-models. These recent findings for isothermal turbulent jets justify the need of more research into intermittency with and without combustion, since the physical process should contain even more complex mechanisms with the addition of heat release. Despite various investigations on the physics of turbulent entrainment in turbulent jets little work has been done on intermittency of turbulent swirl jets with and without combustion.

In recent years considerable advances have been made toward modelling of swirl stabilised turbulent combustion using the large eddy simulation (LES) technique ranging from simple jet flames to complex practical engineering configurations. For example, for gas turbine type combustion applications, Huang et al. (2003) carried out LES for combustion dynamics of a gas turbine swirl injector and later extended their work for bifurcation of flame structures in a 
lean-premixed swirl stabilised combustor (Huang and Yang, 2004). Pierce and Moin (2004) also performed LES of swirling flames. Di Mare et al. (2004) and Kim and Syred (2004) studied LES of model swirl combustors while Selle et. al. (2004) performed LES for an industrial gas turbine burner. Lu et al. (2005) calculated swirl injector flow dynamics in a dump chamber using LES and Grinstein and Fureby (2005) also performed LES for one of the General Electric swirl combustors. Later, Mahesh et al. (2006) carried out LES for a section of a Pratt and Whitney combustor and Garcia-Villalba et. al. (2006) also identified various coherent structures in the near field of swirling flows using LES. Moreover, Wang et al. (2007) performed LES of swirl injector flow dynamics of a General Electric combustor configuration and Boudier et al. (2008) conducted LES calculations of isothermal and reacting flows in a simplified ramjet combustor. Recently Huang and Yang (2009) summarised detailed LES investigation for dynamics and stability of lean-premixed swirl combustion.

Despite the broad range of LES investigations that have been carried for complex swirling flames, identification and analysis of intermittent characteristics for turbulence and scalar mixing are not well understood. Although direct numerical simulation (DNS) is a useful technique to investigate intermittency, it has obvious limitations when considering complex swirling flames due to high Reynolds number with additional complexity due to geometry. Therefore the work described in this paper represents an investigation of the effects of swirl on external intermittency (turbulent-nonturbulent behaviour) characteristic of turbulent unconfined non-premixed swirling flames using LES. The objective is to examine the effects of swirl on the intermittency characteristics of axial velocity, mixture fraction and temperature at important regions such as inside a bluff-body stabilised recirculation zone, 
between two recirculation zones, inside a vortex breakdown bubble and in the outer region of the vortex breakdown bubble in swirling non-premixed flames.

The swirl burner used in this work is the Sydney burner (Al-Abdeli and Masri, 2003, Kalt et al. 2002, Masri et al. 2004), which is frequently used in modelling unconfined swirling flames. Our previous LES investigations targeted some of the Sydney flames for LES validation (Malalasekera et al. 2007, 2008) and instability analysis of swirling flames (RangaDinesh et al. 2009). This work selected an experimental pure methane swirling flame (SM1) with swirl number 0.5 as a base while generating another two numerical flames with increased swirl numbers 0.75 and 1.0. The paper is organised as follows: Section 2 describes the swirl burner experimental details, followed by the theoretical formulations and modelling in section 3 . In section 4 we discuss the numerical computation followed by the results and discussion in section 5 which focuses on the probability density functions (pdfs) and radial profiles of intermittency for velocity, mixture fraction and temperature behaviour for different swirl numbers. We summarise the conclusions in section 6 and suggest future work.

\section{Swirl Burner and Flame Conditions}

A schematic diagram of the experimental burner is given in Figure 1 (Kalt et al. 2002, AlAbdeli and Masri, 2003, Masri et al. 2004). The burner has a fuel jet of diameter $3.6 \mathrm{~mm}$ surrounded by a bluff body of $\mathrm{D}=50 \mathrm{~mm}$ diameter. The burner also has a primary annulus $5 \mathrm{~mm}$ wide around the bluff body which provides both axial and swirling air. Swirl is introduced aerodynamically by using tangential ports $300 \mathrm{~mm}$ upstream of the burner exit. The burner is installed in a wind tunnel which provides a co-flow secondary air stream. In our computations we assumed $\mathrm{x} \mathrm{mm}$ as axial distance and $\mathrm{r} \mathrm{mm}$ as radial distance. 
The swirl number is defined as the ratio between the axial flux of the swirl momentum, $G_{\phi}$ $\left(\mathrm{kgm}^{2} \mathrm{~s}^{-2}\right)$, to the axial flux of the axial momentum $G_{x}\left(\mathrm{kgm}^{2} \mathrm{~s}^{-2}\right)$ multiplied by a characteristic length $R(\mathrm{~m})$. Here we take the radius of the swirl annulus as the characteristic radius. The swirl number is given by:

$$
S=\frac{G_{\phi}}{R G_{x}}=\frac{\int_{0}^{R} U W r^{2} d r}{R \int_{0}^{R} U^{2} r d r}
$$

Where $<U>m / s$ and $<W>m / s$ are the mean axial and tangential velocities at the exit plane, and $R(m)$ a characteristic length. However, the flow in the present burner is describing in terms of the geometric swirl number $S_{g}$, which is defined as the ratio between bulk tangential to bulk axial velocity. The geometric swirl number $S_{g}$ is linearly proportional to actual swirl number $S$ (Al-Abdeli and Masri, 2003). The Reynolds numbers for the fuel jet and primary annulus is defined as $\operatorname{Re}_{j}=U_{j} \times d_{j} / v$ and $\operatorname{Re}_{j}=U_{s} \times d_{s} / v$, where $d_{j}$ is the diameter of the fuel inlet, $U_{j}$ the bulk jet velocity, $U_{s}$ the annulus bulk axial velocity and $r_{s}$ the outer radius of the annulus (Al-Abdeli and Masri, 2003). The experimental variables were the fuel jet velocity $U_{j}$, the bulk axial and tangential velocities $U_{s}$ and $W_{s}$ of the primary air stream, and the mean co-flow velocity $U_{e}$ of the secondary air stream.

In the present study only the pure methane SM1 flame from the experimental data base (Kalt et al. 2002, Al-Abdeli and Masri, 2003, Masri et al. 2004) is considered, which has a fuel jet velocity of $32.7 \mathrm{~m} / \mathrm{s}$ with a swirl number $\mathrm{S}=0.5$. The flame contains two recirculation zones, the first closer to the bluff body which stagnates at about $43 \mathrm{~mm}$ and the second further downstream which stagnates at $70 \mathrm{~mm}$ on the centreline. The mean stoichiometric mixture 
fraction occurs on the centreline at an axial position that is consistent with the visible length of the flame (0.12m) (Masri et al. 2004). In addition to the SM1 flame, two more numerical flames have been considered and defined as SM1a and SM1b with corresponding swirl numbers 0.75 and 1.0 respectively. Table 1 shows the simulated parameters for the three cases.

\begin{tabular}{|l|l|l|l|l|l|l|l|}
\hline Case & Fuel & $U_{j}(m / s)$ & $U_{s}(m / s)$ & $W_{s}(m / s)$ & $U_{e}(m / s)$ & $S_{g}$ & $\operatorname{Re}_{s}$ \\
\hline SM1 & $\mathrm{CH}_{4}$ & 32.7 & 38.2 & 19.1 & 20.0 & 0.5 & 75,900 \\
\hline SM1a & $\mathrm{CH}_{4}$ & 32.7 & 38.2 & 29.3 & 20.0 & 0.75 & 75,900 \\
\hline SM1b & $\mathrm{CH}_{4}$ & 32.7 & 38.2 & 39.0 & 20.0 & 1.0 & 75,900 \\
\hline
\end{tabular}

Table 1. Details about the characteristics properties of SM1, SM1a, SM1b flame series

\section{Governing equations and Modelling}

The LES calculation consists of solving the temporal development of large scale structures by applying a spatial filter to the governing equations. In this work, we use an implicit box (tophat) filter, which fits naturally into the finite volume formulation. By applying a spatial filter, the filtered governing equations for mass, momentum and mixture fraction can be written as follows:

$$
\frac{\partial \bar{\rho}}{\partial t}+\frac{\partial \bar{\rho} u_{j}}{\partial x_{j}}=0
$$




$$
\begin{aligned}
\frac{\partial \bar{\rho} u_{i}}{\partial t}+\frac{\partial\left(\bar{\rho} u_{i} u_{j}\right)}{\partial x_{j}}=- & \frac{\partial \bar{P}}{\partial x_{i}}+\frac{\partial}{\partial x_{j}}\left[\overline{2 \rho}\left(v+v_{t}\right)\left[\frac{1}{2}\left(\frac{\partial u_{i}}{\partial x_{j}}+\frac{\partial u_{j}}{\partial x_{i}}\right)-\frac{1}{3} \delta_{i j} \frac{\partial u_{k}}{\partial x_{k}}\right]\right] \\
& +\frac{1}{3} \frac{\partial}{\partial x_{j}}\left[\bar{\rho} \delta_{i j} \tau_{k k}\right]+\bar{\rho} g_{i} \\
\frac{\partial \bar{\rho} \tilde{f}}{\partial t}+\frac{\partial}{\partial x_{j}}\left(\bar{\rho} \tilde{u}_{j} \tilde{f}\right)= & \frac{\partial}{\partial x_{j}}\left[\bar{\rho}\left(\frac{v}{\sigma}+\frac{v_{t}}{\sigma_{t}}\right) \frac{\partial \tilde{f}}{\partial x_{j}}\right]
\end{aligned}
$$

Here over-bar is the spatial filter, tilde the Favre filter, $\rho$ the density, $u_{i}$ the velocity component in $x_{i}$ direction, $p$ the pressure, $v$ the kinematics viscosity, $f$ the mixture fraction, $v_{t}$ the turbulent viscosity, $\sigma$ the laminar Schmidt number, $\sigma_{t}$ the turbulent Schmidt number and $\tau_{k k}$ the isotropic part of the sub-grid scale stress tensor. The laminar Schmidt number was set to 0.7 and the turbulent Schmidt number for mixture fraction was set to 0.4.

For unclosed terms in the momentum and mixture fraction equations, the sub grid scale contribution is modelled through the turbulent eddy viscosity $v_{t}$ by applying a Smagorinsky eddy viscosity model (1963):

$v_{t}=C_{s} \bar{\Delta}^{2}\left|S_{i, j}\right|=C_{s} \bar{\Delta}^{2}\left|\frac{1}{2}\left(\frac{\partial \tilde{u}_{i}}{\partial x_{j}}+\frac{\partial \tilde{u}_{j}}{\partial x_{i}}\right)\right|$

Here $C_{s}$ is the model parameter and $\bar{\Delta}$ is the filter width. The model parameter $C_{s}$ is obtained using the localised dynamic procedure of Piomelli and Liu (1995).

In LES, chemical reactions occur at the sub-grid scales and therefore modelling is required for combustion chemistry. Here an assumed probability density function (PDF) for the mixture fraction is chosen as a means of modelling the sub-grid scale mixing with $\beta$ PDF 
used for the mixture fraction. The functional dependence of the thermo-chemical variables is closed through the steady laminar flamelet approach. In this approach the variables such as density, temperature and species concentrations depend on Favre filtered mixture fraction, mixture fraction variance and scalar dissipation rate. The sub-grid scale variance of the mixture fraction is modelled using the gradient transport model. The flamelet calculations were performed using the Flamemaster code developed by Pitsch (1998), which incorporates the GRI 2.11 mechanism with detailed chemistry (2006).

In laminar flamelet model, the mixture fraction and the non-equilibrium parameter scalar dissipation rate are the two key parameters, which determine the thermochemical composition of the turbulent flame. In flamelet approach the joint probability density function (PDF) $P(f, \chi)$ of mixture fraction $f$ and scalar dissipation rate $\chi$ is used to determine the filtered values of temperature, density and species mass fractions. Thus the filtered value of the scalar variable is given by

$$
\phi=\int_{0}^{\infty} \int_{0}^{1} \phi(f, \chi) P(f, \chi) d f d \chi
$$

According to the flamelet model formulations, it is assumed that the mixture fraction and the scalar dissipation rate are statistically independent. Thus the joint PDF can be decomposed into two parts such that

$$
\phi=\int_{0}^{\infty} \int_{0}^{1} \phi(f, \chi) P(f) P(\chi) d f d \chi
$$


Here we describes an assumed probability density function (PDF) for the mixture fraction is as a means of modelling the sub-grid scale mixing with $\beta$ probability density function (PDF) used for the mixture fraction. The two independent PDFs can be written as

$$
\begin{aligned}
& P(f)=\frac{\Gamma\left(\beta_{1}+\beta_{2}\right)}{\Gamma\left(\beta_{1}\right) \Gamma\left(\beta_{2}\right)} f^{\beta_{1}-1}(1-f)^{\beta_{2}-1} \\
& P(\chi)=\frac{1}{\chi \sigma \sqrt{2 \pi}} \exp \left(-\frac{1}{2 \sigma^{2}}(\ln (\chi)-\mu)^{2}\right)
\end{aligned}
$$

Where

$$
\begin{aligned}
& \beta_{1}=f\left[\frac{f(1-f)}{f^{2}}-1\right] \\
& \beta_{2}=\frac{(1-f)}{f} \beta_{1}
\end{aligned}
$$

Here the filtered mixture fraction variance $f^{22}$ and filtered scalar dissipation rate $\chi$ still remain an unknown quantities and have to be modelled. The mixture fraction variance and the scalar dissipation rate are modelled using the model equations:

$$
\begin{aligned}
& f^{22}=C \bar{\Delta}^{2}\left(\frac{\partial f}{\partial x_{j}} \frac{\partial f}{\partial x_{j}}\right) \\
& \chi=2\left(\frac{v}{\sigma}+\frac{v_{t}}{\sigma_{t}}\right)\left(\frac{\partial f}{\partial x_{j}} \frac{\partial f}{\partial x_{j}}\right)
\end{aligned}
$$

Where $C \approx 0.1$ has proved successful (Di Mare et al. 2004) and $\bar{\Delta}=\left(\bar{\Delta}_{x} \bar{\Delta}_{y} \bar{\Delta}_{z}\right)^{\frac{1}{3}}$. 


\section{Numerical Computations}

The resultant governing equations and boundary conditions are solved numerically by means of a pressure based finite volume methodology on a Cartesian coordinate system. All simulations were performed using the LES code PUFFIN originally developed by Kirkpatrick et al. (2003) and later extended by Ranga Dinesh (2007, 2009). Second order central differences (CDS) are used for the spatial discretisation of all terms in both the momentum equation and the pressure correction equation. This minimises the projection error and ensures convergence in conjunction with an iterative solver. The diffusion terms of the mixture fraction transport equation are also discretised using the second order CDS. However, the convection term in the mixture fraction transport equation is discretised using a Simple High Accuracy Resolution Program (SHARP) developed by Leonard (1987).

An iterative time advancement scheme is used for variable density calculation. First, the time derivative of the mixture fraction is approximated using the Crank-Nicolson scheme. The flamelet library yields the density and calculate filtered density field at the end of the time step. The new density at this time step is then used to advance the momentum equations. The momentum equations are integrated in time using a second order hybrid scheme. Advection terms are calculated explicitly using second order Adams-Bashforth while diffusion terms are calculated implicitly using second order Adams-Moulton to yield an approximate solution for the velocity field. Finally, mass conservation is enforced through a pressure correction step in which the approximate velocity field is projected onto a subspace of divergence free velocity field. Several outer iterations (8-10) are used to achieve the convergence for each time step and time steps are advanced with variable Courant number in the range of 0.3-0.6. The Bi Conjugate Gradient Stabilized (BiCGStab) method with a Modified Strongly Implicit (MSI) 
preconditioner is used to solve the system of algebraic equations resulting from the discretisation.

All three simulations for flames SM1, SM1a and SM1b were performed on non-uniform Cartesian grids with the dimension of $300 \times 300$ x 250mm by employing 150x150x150 grid points in $\mathrm{x}, \mathrm{y}$ and $\mathrm{z}$ directions respectively giving a total number of approximately 3.4 million cells. The non-equidistant grid is refined to better resolve the fuel jet, the primary annulus and the bluff-body wall. For this work, grid lines in $\mathrm{x}$ - and $\mathrm{y}$ - directions used an expansion ratio of $\gamma_{x y}=\Delta x(i) / \Delta x(i-1)=1.05$ and an expansion ratio of $\gamma_{z}=1.06$ was used in the zdirection.

The inlet mean velocity profiles were generated from

$<U>=1.218 U_{j}\left(1-\frac{|r|}{1.01 . r_{j}}\right)^{1 / 7}$

Where $U_{j}$ is the bulk velocity, $\mathrm{r}$ the radial distance from the jet centreline and $r_{j}$ the fuel jet radius of $1.8 \mathrm{~mm}$. The coefficient 1.28 is set to obtain the correct massflow rate at the inlet and the scale 1.01 is introduced to ensure that velocity gradient is finite at the walls. A similar format is used for the primary annulus with $U_{j}$ replaced by the bulk axial velocity $U_{s}$ and bulk swirling velocity $W_{s}$ and $r$ being the radial distance from the centre of the primary annulus.

The velocity fluctuations for both axial and swirl components were generated by a Gaussian random number generator and are then added to the mean velocity profiles. A top hat profile is used as the inflow condition for the mixture fraction. A free slip boundary condition is 
applied at the solid walls and at the outflow, a convective boundary condition is used for the velocities and a zero normal gradient boundary condition is used for the mixture fraction. Simulations were performed for a time sufficient to achieve convergence before storing data for the intermittency and time-averaged calculations.

\section{Results and Discussion}

This section presents a detailed description of the computed pdf distributions and radial variation of the external intermittency fields for the velocity, mixture fraction and temperature for three different flames. The considered flames are SM1, SM1a and SM1b with swirl numbers $0.5,0.75$ and 1.0 respectively. The swirling flames considered in this work for the intermittency investigation exhibit complex flow structures such as two recirculation zones (bluff body stabilised and swirl induced), a collar like flow structure and also centre jet precession behaviour. The intention is to study the effects of swirl on turbulent intermittency and scalar mixing in the presence of precession, recirculation and vortex breakdown aiming for further improvement in determining more local properties of intermittency such as the characteristics and scales of fluid dynamics and scalar mixing for swirl stabilised turbulent combusting flows.

\section{Intermittency:}

In general, there are two main streams of research that treat intermittency in present day modelling efforts of turbulent combustion: external intermittency and internal intermittency. Both external and internal intermittency can be seen as multiscale spatio temporal random processes. However, external intermittency is quite different from the internal intermittency and their multi-scale and multi physics characteristics make it difficult to model as a one 
physical problem for complex turbulent combustion problems. Therefore isolation treatments are the best possible ways to tackle local characteristics of external intermittency in turbulent combustion explicitly or implicitly. The interfacial distinction between turbulence-bearing fluid (e.g. the jet or the boundary layer) and non-turbulent fluid (free stream) is referred to as the external intermittency. Internal intermittency refers to local fluctuations of turbulence intensity (the intermittency in an inertial range of a turbulent flow). Mathematically external intermittency can be expressed using an indicator function with the value of one in turbulent regions and zero in non-turbulent (laminar) regions. The indicator function represents the fraction of the time interval during which a point is inside the turbulent fluid.

Various methods are available to determine the external intermittency factor $(\gamma)$ in a heated flow for different variables such as velocity, passive and active scalars (Bilger et al. 1976, Schefer and Dibble, 2001). The following section provides a discussion of the external intermittency calculation procedure used in this work. The most common method is to estimate a pdf by computing a normalised histogram. This method assumes that the pdf is smooth at the scale of one histogram bin. By applying this procedure, the pdfs were calculated from no less than 4000 measurements at each spatial location using 50 bins equally spaced over the $3-\sigma$ limits of the data. The distributions are normalised such that

$$
\int_{0}^{1} P(f) d f=1
$$

Therefore the external intermittency for velocity, mixture fraction and temperature can be defined as the fraction of time that the variable value is greater than an arbitrary threshold value. The corresponding intermittency is calculated from the probability density distribution 
of the instantaneous values. For example if a threshold value of $f_{\text {th }}$ for variable $f$ is selected, the area under the probability density distribution relates to the intermittency such that:

$\gamma=P\left(f>f_{t h}\right)$

\section{LES snapshots and selected flame regions:}

Figures 2-4 show snapshots of the flame temperature for SM1, SM1a and SM1b respectively. All three flames show high temperature regions on the boundary of the first bluff body stabilised recirculation zone and further downstream near the centreline region inside the second downstream recirculation zone. The small neck region is visible for the SM1 flame near $\mathrm{x}=60 \mathrm{~mm}$ (downstream from the burner exit plane) which has also been observed in the experimental data (Kalt et al 2002). Moreover, all three flames show stagnation regions in the upstream first recirculation zone, where the mean axial velocity is zero just above $40 \mathrm{~mm}$, and for the downstream second recirculation zone, where the axial velocity on the centreline is below zero around $x=70-130 \mathrm{~mm}$ depending on the strength of the swirl. Here we focus on four important axial locations and produce pdf and radial intermittency profiles for velocity, mixture fraction and temperature. The first axial location is selected inside the bluff body stabilised recirculation zone $(\mathrm{x}=30 \mathrm{~mm})$, the second location is situated between the upstream and downstream recirculation zones $(x=55 \mathrm{~mm})$, the third axial location is inside the downstream recirculation zone also known as the vortex breakdown bubble $(\mathrm{x}=100 \mathrm{~mm})$ and the fourth axial location is further downstream and on the boundary of the downstream recirculation zone $(\mathrm{x}=155 \mathrm{~mm})$. The selected axial locations are marked in each figure. The pdfs for velocity, mixture fraction and temperature at each axial location $(x=30,55,100$, $155 \mathrm{~mm})$ are generated for equal radial distances $(\mathrm{r}=0,12,24,32 \mathrm{~mm})$. The following three 
sections discuss the pdf and intermittency for velocity, mixture fraction and temperature respectively.

\section{Validation studies:}

Before discussing the intermittency results of this study, the accuracy of the current LES results should be addressed. Here we consider the experimental data of flame SM1 (Kalt et al. 2002, Al-Abdeli and Masri, 2003) to validate our numerical results obtained from the LES calculations.

The comparison between LES results and experimental measurements for the time averaged mean axial (left side) and mean swirl (right side) velocity at $\mathrm{x} / \mathrm{D}=0.136,0.8,1.4$ and 2.5 is shown in Figure 5. Where $\mathrm{D}=50 \mathrm{~mm}$ is the diameter of the bluff body and $\mathrm{R}=25 \mathrm{~mm}$ is the radius of the bluff body. To further demonstrate the recirculation zones, Figure 6 shows the contour plot of LES time-averaged mean axial velocity. The LES predictions captured a relatively short bluff body stabilised recirculation zone and also a second downstream central recirculation zone (vortex breakdown bubble) as observed by the experimental measurements (Kalt et al. 2002). LES calculations further indicate that the axial and radial extents of the bluff body stabilised recirculation zone and swirl induced central recirculation zones are in close agreement with experimental observations. Despite a slight over prediction at $\mathrm{x} / \mathrm{D}=0.136$ and 1.4 , the mean swirling velocities also agree well with the experimental measurements. The over prediction may be attributed to the shear layer instability of the upstream recirculation zone and centre jet precession. However, the overall agreement between experimental and simulated time averaged mean axial and swirl velocities are reasonably good at all considered axial locations. Shown in Figure 7 is the time averaged axial (left side) rms velocity and swirling (right side) rms velocity. The calculated rms axial 
velocity slightly underestimates at $\mathrm{x} / \mathrm{D}=1.4$ and $\mathrm{rms}$ swirl velocity overestimates at $\mathrm{x} / \mathrm{D}=0.8$ and 1.2. Our previous LES study has shown that the centre jet of flame SM1 appears to move more into one side of the geometric centreline before crossing over to the other side. Hence the centre jet has an irregular random motion which cannot be defined as regular time periodic behaviour (Ranga Dinesh et al. 2010). In addition, the occurrence of bluff-body stabilised recirculation zone may also cause some vortex shedding and thus small discrepancies are apparent. Despite the small discrepancies, the comparison between LES calculations and experimental measurements for the rms velocities are good at most axial locations.

Figure 8 shows the time averaged mean mixture fraction (left side) and mean temperature (right side) at $\mathrm{x} / \mathrm{D}=0.2,0.8$ and 1.5 . The LES mean mixture fraction under predicts at $\mathrm{x} / \mathrm{D}=0.8$ and over predicts on the centreline at $\mathrm{x} / \mathrm{D}=1.5$. This is exactly the stagnation region of the central recirculation zone where present LES may not have captured the correct mixture fraction distribution in this highly dynamic region. Although the mixture fraction predictions are reasonably good at locations $\mathrm{x} / \mathrm{D}=0.2$ and 0.8 the predicted peak temperature at the outer shear layer at these axial locations is somewhat over-predicted. The flame may be subjected high shear effects in this region and the use of a steady flamelet model to extract thermochemical properties may not be perfectly valid in this region, which could have resulted in these discrepancies. However, the overall agreement between computations and measurements for the time averaged mean mixture fraction and mean temperature are good and the calculations predict the trends which appeared in the temperature field. This validation study shows good agreement with experimental measurements which promotes confidence in presenting the effects of swirl on velocity and scalar intermittency. The 
simulation results are analysed using the instantaneous LES base aiming to demonstrate the external intermittency of swirling flames.

\section{Velocity intermittency:}

Figures 9-12 show the pdf of axial velocity at $x=30,55,100$ and $155 \mathrm{~mm}$ respectively. The pdfs of axial velocity for all three swirl numbers have a similar shape near the centreline region $(\mathrm{r}=0 \mathrm{~mm}, 12 \mathrm{~mm})$ at $\mathrm{x}=30 \mathrm{~mm}$. Since the circular bluff body at the inlet forms a near field recirculation zone for all three cases, the effect of swirl is minimal near the centreline region given the fact that the swirl has been introduced from the secondary inlet. However at far radial locations (the outer region of the bluff body stabilised recirculation zone) $(r=24 \mathrm{~mm}$ and $r=32 \mathrm{~mm}$ ) the pdf shapes start to deviate from each other due to direct effect of tangential velocity. The pdfs of velocity at $\mathrm{x}=55 \mathrm{~mm}$ (Figure 10) again show similar shapes on the centreline and start to deviate at far radial locations. It is important to note that both LES and experimental results showed that the centre jet has an irregular random motion. They also showed a large scale wobbling motion of the jet tip in flame SM1. Since the centre jet extends axially closer to $x=30 \mathrm{~mm}$, the pdfs of velocity on the centreline at $\mathrm{x}=30 \mathrm{~mm}$ show a non-Gaussian behaviour $(\mathrm{r}=0 \mathrm{~mm})$ due to the direct impact of the wobbling motion of the centre jet tip. However, all figures at remaining axial locations ( $\mathrm{x}=55,100,155 \mathrm{~mm})$ show that the pdfs near the centreline follow the Gaussian shape and then move to a delta function at far radial locations. Moreover, close to the centre-line $(\mathrm{r}=0,12 \mathrm{~mm})$ these distributions are relatively broad and generally Gaussian, whereas with increasing radial distance they narrow and ultimately form spikes on the co-flow velocity $(\mathrm{r}=32 \mathrm{~mm})$. The pdfs of the axial velocity at far radial locations $(\mathrm{r}=24 \mathrm{~mm}$ and $32 \mathrm{~mm}$ )at all four axial positions show rapid changes mainly due to the instability occurring on the boundaries of both the upstream recirculation 
zone and the downstream vortex bubble. Since both the width and length of the recirculation zones depend on swirl strength, the highest swirl case has a wider pdf compared with the lower swirl case (Figures 11 and 12).

Figure 13 shows the radial profiles of the axial velocity intermittency at $x=30,55,100$ and $155 \mathrm{~mm}$ respectively. Since the burner has a secondary co-flow velocity of $20 \mathrm{~m} / \mathrm{s}$, the calculation used a threshold value of $u_{t h}=10 \mathrm{~m} / \mathrm{s}$ (half of the secondary axial co-flow velocity). This has been further supported by the vortex breakdown (VB) phenomenon for all three cases. The formation of the centre recirculation zone (VB bubble) creates a negative axial velocity near the centreline region. Therefore using a lower threshold value $(10 \mathrm{~m} / \mathrm{s})$ compared to the inlet axial velocity $(32.7 \mathrm{~m} / \mathrm{s})$ flow conditions should demonstrate the effect of swirl on intermittency of the axial velocity near the centreline region due to the axial extends of negative axial velocity (recirculation and vortex breakdown) depend on the strength of swirl. However, it is important to note that the shape of the external intermittency distribution for the axial velocity component is more sensitive to threshold value, especially with the addition of swirl due to formation of the negative axial velocity region on the centreline compared to a standard round jet where the intermittency profile of the axial velocity should follow a standard Gaussian distribution.

The distributions of the velocity intermittency profiles at different axial locations indicate the effect of swirl on external intermittency with respect to a selected threshold value. As expected, differences in the axial velocity intermittency appear mainly near the centreline region. For example, Figure 13 shows little effect of swirl on the velocity intermittency near the centreline inside the bluff body stabilised recirculation zone $(x=30 \mathrm{~mm})$. However, the effect of swirl on intermittency of the axial velocity component is apparent at other axial 
locations such as between the two recirculation regions $(x=55 \mathrm{~mm})$, inside the second recirculation region $(\mathrm{x}=100 \mathrm{~mm})$ and at the downstream boundary of the second recirculation region $(x=155 \mathrm{~mm})$. The effect of swirl on axial velocity intermittency also indicates the presence of a small scale turbulent fluctuation near the centreline region which is important in swirl combustion. The radial profiles of velocity intermittency on the other hand follow a similar distribution for all three cases at far radial positions at the considered axial locations. This is expected due to the co-flow velocity of $20 \mathrm{~m} / \mathrm{s}$ which is twice the considered threshold value $(10 \mathrm{~m} / \mathrm{s})$ for determining the velocity intermittency.

\section{Mixture fraction intermittency:}

In Figures 14-17 the pdfs of the mixture fraction are displayed at axial locations $x=30,50$, 100 and $155 \mathrm{~mm}$ respectively. These results are qualitatively similar to the velocity pdfs. The centreline mixture fraction pdfs at $\mathrm{x}=30 \mathrm{~mm}$ (Figure 14) show non-Gaussian behaviour for all three cases due to centre jet precession which has been observed in both LES and experimental data. However, the shape of the pdfs at other axial locations ( $x=55,100$ and $155 \mathrm{~mm})$ show Gaussian behaviour on the centreline $(\mathrm{r}=0 \mathrm{~mm})$ and near centreline radial locations $(\mathrm{r}=12 \mathrm{~mm})$ before reverting into delta functions at far radial locations $(\mathrm{r}=32 \mathrm{~mm})$ (Figures 15, 16 and Figure 17). Furthermore the pdfs show a wider spread of the mixture fraction field at far radial locations $(\mathrm{r}=24,32 \mathrm{~mm})$ for the highest swirl case due to higher centrifugual force for all four axial locations. Figure 18 shows the radial profiles of mixture fraction intermittency at $\mathrm{x}=30,55,100$ and $155 \mathrm{~mm}$ respectively. The relation between the stoichiometric mixture fraction and temperature is an important factor in this work as a result of using the flamelet model. Therefore selecting a stoichimetric mixture fraction value as a threshold could help to determine the similarities and differences between the mixture 
fraction and temperature intermittency. Here we used a threshold value of $f_{\text {th }}=0.054$ which is a stoichiometric mixture fraction for pure methane SM1 swirling flame (Kalt et al. 2002, Al-Abdeli and Masri, 2003). As seen in Figure 18 the radial variation of the mixture fraction intermittency follows a Gaussian shape at all axial locations for all three cases. Since absolute values of intermittency are very similar at many radial locations for all three cases, we conclude that the swirl impact on external intermittency is minimal for the first three axial locations $(x=30,55,100 \mathrm{~mm})$. However, as seen in Figure 18 the swirl starts to affect the mixture fraction intermittency at the furthest downstream axial location $(x=155 \mathrm{~mm})$ depending on the tangential velocity strength.

\section{Temperature intermittency:}

The pdfs of temperature at $x=30,55,100$ and $155 \mathrm{~mm}$ are shown in Figures 19-22. Although the time averaged mean temperature field for the SM1 flame shows good agreement with the experimental data, the instantaneous pdfs of temperature show an interesting variation from the centreline to far radial locations and thus indicate the presence of temperature intermittency for certain regions of the flame. Again, similar to velocity and mixture fraction, the temperature field follows a non-Gaussian shape on the centreline at $\mathrm{x}=30 \mathrm{~mm}$ (Figure 19). However, at $\mathrm{x}=55 \mathrm{~mm}$ the pdf of temperature follows a Gaussian shape on the centreline and then changes to a delta function at far radial locations. The effect of swirl is also apparent at far radial locations as the temperature pdf shows an intermediate shape between a Gaussian and delta function at both $\mathrm{r}=24 \mathrm{~mm}$ and $32 \mathrm{~mm}$ for high swirl number $(\mathrm{S}=1.0)$. It is also important to note that the small variation of mixture fraction could lead to a variation of temperature as a result of using the steady laminar flamelet model. Therefore the pdf variation of mixture fraction is directly linked to the pdf of temperature and thus forma a non- 
Gaussian shape. Additionally increasing the swirl number affects the flame temperature in a region close to the centreline $(\mathrm{r}=0 \mathrm{~mm}$ and $12 \mathrm{~mm})$ and also at far radial locations $(\mathrm{r}=24 \mathrm{~mm})$.

Figure 23 shows the radial variation of temperature intermittency at $\mathrm{x}=30,55,100$ and $155 \mathrm{~mm}$ respectively. Here we have used a threshold value of $t_{h}=750 \mathrm{~K}$ which is approximately half the value of the maximum mean temperature calculated from the simulation. As seen in Figure 23, the centreline intermittency values are less than unity and thus indicate reduce temperature with respect to a threshold temperature value for all three cases. This has been observed inside the upstream recirculation zone where turbulence can play a vital role in swirl stabilised combustion with the presence of counter rotating vortices. However, the temperature on the centreline and near the centreline at other axial locations $(\mathrm{x}=55,100,155 \mathrm{~mm})$ is slightly increased compare to $\mathrm{x}=30 \mathrm{~mm}$ as intermittency values are unity corresponding to the threshold for several radial locations near the centreline region before starting to decay at far radial distances. In addition the temperature intermittency also depends on rapid changes of the velocity fluctuations due to its relation with the mixture fraction variance.

\section{Conclusions}

A large eddy simulation has been applied to study the effect of swirl on intermittency of turbulent non-premixed flames. Probability density functions and intermittency profiles have been generated for velocity, mixture fraction and temperature. Derived probability density functions show changes from a Gaussian shape to a delta function with increased radial distance at several selected axial locations. Non-Gaussian behaviour of both velocity and scalar pdfs were observed on the centreline as a result of centre jet precession and the precessing vortex core. The precession motion of the centre jet not only affects the region 
close to the jet, but also the downstream region in the free swirl jet. The differences of the pdfs and intermittency in velocity and scalars for a complex swirling flame in the presence of recirculation zones and precession motion demonstrate the effect of swirl on external intermittency. The entrainment process of sheared fluid in the flame is governed by the large scale motions of turbulent eddies enclosing irrotational fluid, so the radial transport of momentum and scalar quantities in such a free shear flow is strongly dependent on intermittency phenomena, and the explicit derivation of external intermittency is thus important for accurate prediction of momentum exchange. Further extraction of the characteristic time and length scales, and velocities, of the entrained irrotational fluid could allow quantitative estimation of the influence of intermittency on this momentum exchange.

We have discussed the intermittent nature of turbulent swirling flames using a localised dynamic LES subgrid turbulence model and steady laminar flamelet combustion model. However, using a steady laminar flamelet model might restrict the combustion physics of intermittent behaviour especially at some regions of the swirling flames. More advanced flamelet based models such as the unsteady flamelet model and the flamelet progress variable approach may be useful for identifying intermittency of the scalar dissipation rate (unsteady strains) and thus the temperature of complex swirling flames.

Despite using the dynamic sub-grid scale model, there remains a requirement for improved sub-grid scale modelling for use with LES in high Reynolds number swirl stabilised combusting flows. Recent experimental and numerical findings of Pedersen et al. (2005, 2009) and Da Silva (2009) further support the present findings especially with regards to tackling the behaviour of the turbulent-nonturbulent interface in unconfined turbulent jets and swirling flames. The findings of Pedersen et al. (2005) further highlight the fact that eddy 
viscosity has a nonzero and constant value in the irrotational outer flow region and thus leads to a conclusion that sub-grid turbulence models should be developed to cope with the strong inhomogeneity of the flow near the edge of the jet. This should be even more complex for swirling flows due to high shear effects between different flow regions. The coupling relation between intermittency-vortex breakdown and precession and their combined effect on complex combustion characteristics such as local extinction in swirl stabilised turbulent flame need further attention. Therefore more investigations should be carried out to determine relations between intermittency and the rate of turbulent and molecular mixing rates for complex and practically relevant turbulent swirling flames

A number of methods that allow intermittency might be introduced into sub grid scale models. One such method is to extend the dynamic sub-grid scale approach employed in this work to account external intermittency by determining the degree to which the resolved eddy scales are space-filling using similar local box estimation. This might involve evaluating intermittency by comparing simulated estimates with expected (fully turbulent) values for appropriate representative quantities, notably the degree of helicity. Furthermore, the work carried out by Pedersen et al. (2005) showed that turbulent entrainment appears to be caused by small scale eddying motion at the outward propagating interface instead of large scale engulfment. Therefore a fractal based approach that has been used previously for small scale internal intermittency (Sulem et al. 1978) could be used as a comprehensive model to determine the interface dynamics due to external intermittency of complex swirling flames. 


\section{Acknowledgements}

This work was supported by the Engineering and Physical Research Council (EPSRC) under grant number (EP/E036945/1) on the Modelling and Simulation of Intermittent Flows.

\section{References}

Al-Abdeli, Y.M., and Masri, A.R. 2003. Stability characteristics and flow fields of turbulent non-premixed swirling flames. Combust. Theory Model., 7:731-766.

Bilger, R.W., Antonia, R.A., and Sreenivasan, K.R. 1976. Determination of intermittency from the probability density function of a passive scalar. Phys. Fluids, 19:1471-1474.

Boudier, G.L., Gicquel, Y.M., and Poinsot, T.J. 2008. Effects of mesh resolution on large eddy simulations of reacting flows in complex geometries. Combust. Flame, 155:196-214.

Bowman, C.T., Hanson, R.K., Davidson, D.F., Gardiner, Jr., Lissianski, V., Smith, G.P., Golden, D.M. Frenklach, M., and Goldenberg, M. 2006. GRI MECH 2.11.http://www.me.berkeley.edu/gri mech, 11/05/2007.

Byggstoyl, S., and Kollmann, W. 1981. Closure model for intermittent turbulent flows. Int. J. Heat Mass Transfer, 24:1811-1818.

Chevray, R., and Tutu, N.K. 1978. Intermittency and preferential tranpsort of heat in a round jet. J. Fluid. Mech., 88:133-145.

Cho, J.R., and Chung, M.K. 1992. A k-epsilon-gamma equation turbulence model. J. Fluid Mech., 237:301-322.

Corrsin, S., and Kistler, A.L. 1955. Free stream boundaries of turbulent flows. NACA Report, 1244. 
Da Silva, C.B. 2009. The behaviour of the subgrid models near the turbulent/ nonturbulent interface in jets. Phy. Fluids, 21:081702.

DeBruyn, S.M., Riley, J.J., Kosaly, G., and Cook, A.W. 1998. Investigation of modelling for non-premixed turbulent combustion. Flow Turbul. Combust., 60:105-122.

Dewan, A., Arakeri, J.H., and Srinivasan, J. 1997. A new turbulence model for the axisymmetric plane. J. Appl. Math Model., 21:709-719.

DiMare, F., Jones, W., and Menzies, K. 2004. Large eddy simulation of a model gas turbine combustor. Combust. Flame, 137:278-294.

Dopazo, C. 1977. On conditioned averages for intermittent turbulent flows. J. Fluid. Mech., $81: 433-445$.

Escudier, M.P., and Keller, J.J. 1985. Recirculation in swirling flows: a manifestation of vortex breakdown. AIAA J., 23:111-116.

Frohlich, J., Garcia-Villalba, M., and Rodi, W. 2006. Identification and analysis of coherent strucures in the near field of a turbulent unconfined annular swirling jet using large eddy simulation. Phys. Fluids, 18:055103.

Gibbson, J.D., and Doering, C.R. 2003. Intermittency in solutions of the three dimensional Navier-Stokes equations. J. Fluid Mech., 478:227-235.

Grinstein, F.F., and Fureby, C. 2005. LES studies of the flow in a swirl gas combustor. Proc. Combust. Inst., 30:1791-1798.

Gupta, A.K., Lilly, D.G., and Syred, N. 1984. Swirl flows. In Swirl flows. Kent Engl: Abacus.

Huang, Y., Sung, H.G., Hsieh, S.Y., and Yang, V. 2003. Large eddy simulation of combustion dynamics of lean premixed swirl stabilised combustor. J. Prop. Power, 19:782794. 
Huang, Y,. and Yang, V. 2004. Bifurcation of Flame Structures in a Lean-Premixed SwirlStabilized Combustor: Transition from Stable to Unstable Flame. Combust. Flame. 136:383389.

Huang, Y., and Yang, V. 2009. Dynamics and Stability of Lean-Premixed Swirl-Stabilized Combustion, Prog. Energy Combust. Sci., 35:293-364.

Jimenez, J. 2000. Intermittency and cascades. J. Fluid Mech., 409:99-120.

Kalita, K., Dewan, A., and Dass, A.K. 2000. Computation of the turbulent plane plume using the model. J. Appl. Math Model., 24:815-826.

Kalt, P.A.M., Al-Abdeli, Y.M., Masri, A.R., and Barlow, R.S. 2002. Swirling turbulent nonpremixed flames of methane: Flowfield and compositional structure. Proc. Combust. Inst., 29:1913-1919.

Kerstein, A.R. 2002. Turbulence in combustion process: Modelling and challenges. Proc. Combust. Inst., 29:1763-1773.

Kim, W.W., and Syed, S. 2004. Large eddy simulation needs for gas turbine combustor design. AIAA, pages 2004-0331.

Kirkpatrick, M.P., Armfield, S.W., and Kent, J.H. 2003. A representation of curverd boundaries for the solution of the Navier-Stokes equations on a staggered three dimensional Cartesian grid. J. Comput. Phys., 184:1-36.

Klebanoff, P.S. 1955. Characteristics of turbulence in boundary layer with zero pressure gradient. NACA Report, 1247.

Kollamann, W., and Janicka, J. 1982. The probability function of a passive scalar in turbulent shear flows. Phy. Fluids, 25:1755-1769.

Li, Y., and Meneveau, C. 2006. Intermittency trends and lagrangian evolution of nongaussian statistics in turbulent flow and scalar transport. J. Fluid Mech., 558:133-142. 
Leonard, B.P. 1987. Sharp simulation of discontinuities in highly convective steady flow. Technical Report 100240, NASA Tech. Mem.

Libby, P.A. 1975. On the prediction of intermittent turbulent flows. J. Fluid. Mech.,68:273279.

Lucca-Negro, O., and O’Doherty, T. 2001. Vortex breakdown: a review. Prog. Energy Combust. Sci., 27:431-481.

Lu, X.Y., Wang, S.W., Song, H.G., Hsieh, S.Y., and Yang, Y. 2005. Large Eddy Simulations of Turbulent Swirling Flows Injected into a Dump Chamber. J. Fluid Mech. 527:171-195.

Mahesh, K., Constantinescu, G., Iaccarino, G., Apte, S., Ham, F., and Moin, P. 2006. Large eddy simulation of reacting turbulent flows in complex geometries. ASME J.Appl. Mech., 73:374-381.

Malalasekera, W., Ranga Dinesh, K.K.J., Ibrahim, S.S., and Kirkpatrick, M.P. 2007. Large eddy simulation of isothermal turbulent swirling jets, Combust. Sci. Tech. 179, 1481-1525. Malalasekera, W., Ranga Dinesh, K.K.J., Ibrahim, S.S., and Masri, A.R. 2008. LES of recirculation and vortex breakdown in swirling flames. Combust. Sci. Tech., 180:809-832. Masri, A.R., Kalt, P.A.M., and Barlow, R.S. 2004. The compositional structure of swirl stabilised turbulent non-premixed flames. Combust.Flame, 137:1-37.

Nakamura, I., Sakai, Y., and Tsunoda, H. 1989. On conditional statistics of the diffusion field of matter by a point source plume in uniform mean shear flow. JSME Int. J., 32:180-190.

Pedersen, J.M., Westerweel, J., Fukushima, C., and Hunt, J.C.R. 2005. Mechanics of the turbulent/nonturbulent interface of a jet. Phy. Rev. Letters, 95:174501.

Pedersen, J.M., Westerweel, J., Fukushima, C., and Hunt, J.C.R. 2009. Momentum and scalar transport at the turbulent/nonturbulent interface of a jet. J. Fluid Mech., 631:199-230.

Pierce, C.D., and Moin, P. 2004. Progress-variable approach for large eddy simulation of non-premixed turbulent combustion. J. Fluid Mech., 504:73-97. 
Piomelli, U., and Liu, J. 1995. Large eddy simulation of rotating channel flows using a localized dynamic model. Phys. Fluids, 7:839-848.

Pitsch, H. 1998. A C++ computer program for 0-d and 1-d laminar flame calculations. Technical report, RWTH Aachen.

Pitsch, H., and Steiner, H. 2000. Large eddy simulation of a turbulent piloted methane-air diffusion flame(Sandia flame D). Phys. Fluids, 12(10):2541-2554.

Pope, S.B. 1984. Calculation of plane turbulent jet. AIAA J., 22:896-904.

Ranga Dinesh, K.K.J. 2007. Large eddy simulation of turbulent swirling flames. PhD thesis, Loughborough University, UK.

Ranga Dinesh, K.K.J., Jenkins, K.W., Kirkpatrick, M.P., and Malalasekera, W. 2009. Identification and analysis of instability in non-premixed swirling flames using LES. Combust. Theo. Model., 13:947-971.

Ranga Dinesh, K.K.J., Jenkins, K.W., Kirkpatrick, M.P., and Malalasekera, W. 2010. Modelling of instabilities in swirling flames. J. Fuel, 89:10 18.

Schefer, R.W., and Dibble, R.W. 2001. Mixture fraction field in a turbulent non-reacting propane jet. AIAA J., 39:64-72.

Selle, L., Lartigue, G., Poinsot, T., and Koch, R., et al. 2004. Compressible large eddy simulation of turbulent combustion in complex geometry on unstructured meshes. Combust. Flame, 137:489-505.

Smagorinsky, J. 1963. General circulation experiments with the primitive equations, the basic experiment. Mon. Weath. Rev., 91:99-164.

Sreenivasan, K.R. 1985. On the fine scale intermittency of turbulence. J. Fluid Mech., 151:81-103.

Syred, N. 2006. A review of instability and oscillation mechanisms in swirl combustion systems. Prog. Energy Combust. Sci., 32:93-161. 
Syred, N., and Beer, J.M. 1974. Combustion in swirling flows: a review. Combust. Flame, 23:143-201.

Sulem, P.L., Frisch, U., and Nelkin, M. 1978. A simple dynamical model of intermittent fully developed turbulence. J. Fluid Mech., 87:719-736.

Townsend, A.A. 1948. Local isotropy in the turbulent wake of cylinder. Austr. J. Sci. Res., $1: 161-168$.

Wang, S.W., Yang, Y., Hsiao, C.C., Hsieh, S.Y., and Mongia, H. 2007. Large-Eddy Simulations of Gas-Turbine Swirl Injector Flow Dynamics. J. Fluid Mech., 583:99-122.

Wygnanski, I., and Fiedler, H.E. 1969. Some measurements in the self preserving jet. J. Fluid. Mech., 38:577-612. 


\section{Figure Captions}

Figure 1. Schematic drawing of the Sydney swirl burner

Figure 2. Snapshot of SM1 flame temperature

Figure 3. Snapshot of SM1a flame temperature

Figure 4. Snapshot of flame SM1b flame temperature

Figure 5. Comparison of mean axial velocity $\langle\mathrm{U}\rangle \mathrm{m} / \mathrm{s}$ and mean swirling velocity $\langle\mathrm{W}\rangle \mathrm{m} / \mathrm{s}$, Lines represent LES results, and symbols represent experimental measurements.

Figure 6. Contour plot of time-averaged mean axial velocity of flame SM1

Figure 7. Comparison of rms axial velocity $\mathrm{rms}$ (u) $\mathrm{m} / \mathrm{s}$ and $\mathrm{rms}$ swirling velocity rms (w) m/s. Lines represent LES results, and symbols represent experimental measurements.

Figure 8. Comparison of mean mixture fraction $F$ and mean temperature $T(K)$. Lines represent LES results, and symbols represent experimental measurements.

Figure 9. Comparisons of velocity pdfs at $\mathrm{x}=30 \mathrm{~mm}$ at equidistant radial locations $\mathrm{r}=0 \mathrm{~mm}$, $12 \mathrm{~mm}, 24 \mathrm{~mm}$ and $32 \mathrm{~mm}$. Here solid line, dashed line and inverted triangles denote results for swirl numbers $0.5,0.75$ and 1.0 
Figure 10. Comparisons of velocity pdfs at $x=55 \mathrm{~mm}$ at equidistant radial locations $\mathrm{r}=0 \mathrm{~mm}$, $12 \mathrm{~mm}, 24 \mathrm{~mm}$ and $32 \mathrm{~mm}$. Here solid line, dashed line and inverted triangles denote results for swirl numbers $0.5,0.75$ and 1.0

Figure 11. Comparisons of velocity pdfs at $\mathrm{x}=100 \mathrm{~mm}$ at equidistant radial locations $\mathrm{r}=0 \mathrm{~mm}$, $12 \mathrm{~mm}, 24 \mathrm{~mm}$ and $32 \mathrm{~mm}$. Here solid line, dashed line and inverted triangles denote results for swirl numbers $0.5,0.75$ and 1.0

Figure 12. Comparisons of velocity pdfs at $x=155 \mathrm{~mm}$ at equidistant radial locations $r=0 \mathrm{~mm}$, $12 \mathrm{~mm}, 24 \mathrm{~mm}$ and $32 \mathrm{~mm}$. Here solid line, dashed line and inverted triangles denote results for swirl numbers $0.5,0.75$ and 1.0

Figure 13. Radial profiles of velocity intermittency at $\mathrm{x}=30 \mathrm{~mm}, 55 \mathrm{~mm}, 100 \mathrm{~mm}$ and $155 \mathrm{~mm}$. Here circles, squares and inverted triangles denote results for swirl numbers $0.5,0.75$ and 1.0

Figure 14. Comparisons of mixture fraction pdfs at $x=30 \mathrm{~mm}$ at equidistant radial locations $\mathrm{r}=0 \mathrm{~mm}, 12 \mathrm{~mm}, 24 \mathrm{~mm}$ and $32 \mathrm{~mm}$. Here solid line, dashed line and inverted triangles denote results for swirl numbers $0.5,0.75$ and 1.0

Figure 15. Comparisons of mixture fraction pdfs at $x=55 \mathrm{~mm}$ at equidistant radial locations $\mathrm{r}=0 \mathrm{~mm}, 12 \mathrm{~mm}, 24 \mathrm{~mm}$ and $32 \mathrm{~mm}$. Here solid line, dashed line and inverted triangles denote results for swirl numbers $0.5,0.75$ and 1.0 
Figure 16. Comparisons of mixture fraction pdfs at $x=100 \mathrm{~mm}$ at equidistant radial locations $\mathrm{r}=0 \mathrm{~mm}, 12 \mathrm{~mm}, 24 \mathrm{~mm}$ and $32 \mathrm{~mm}$. Here solid line, dashed line and inverted triangles denote results for swirl numbers $0.5,0.75$ and 1.0

Figure 17. Comparisons of mixture fraction pdfs at $x=155 \mathrm{~mm}$ at equidistant radial locations $\mathrm{r}=0 \mathrm{~mm}, 12 \mathrm{~mm}, 24 \mathrm{~mm}$ and $32 \mathrm{~mm}$. Here solid line, dashed line and inverted triangles denote results for swirl numbers $0.5,0.75$ and 1.0

Figure 18. Radial profiles of mixture fraction intermittency at $x=30 \mathrm{~mm}, 55 \mathrm{~mm}, 100 \mathrm{~mm}$ and $155 \mathrm{~mm}$. Here circles, squares and inverted triangles denote results for swirl numbers 0.5 , 0.75 and 1.0

Figure 19. Comparisons of temperature pdfs at $x=30 \mathrm{~mm}$ at equidistant radial locations $\mathrm{r}=0 \mathrm{~mm}, 12 \mathrm{~mm}, 24 \mathrm{~mm}$ and $32 \mathrm{~mm}$. Here solid line, dashed line and inverted triangles denote results for swirl numbers $0.5,0.75$ and 1.0

Figure 20. Comparisons of temperature pdfs at $x=55 \mathrm{~mm}$ at equidistant radial locations $\mathrm{r}=0 \mathrm{~mm}, 12 \mathrm{~mm}, 24 \mathrm{~mm}$ and $32 \mathrm{~mm}$. Here solid line, dashed line and inverted triangles denote results for swirl numbers $0.5,0.75$ and 1.0

Figure 21. Comparisons of temperature pdfs at $\mathrm{x}=100 \mathrm{~mm}$ at equidistant radial locations $\mathrm{r}=0 \mathrm{~mm}, 12 \mathrm{~mm}, 24 \mathrm{~mm}$ and $32 \mathrm{~mm}$. Here solid line, dashed line and inverted triangles denote results for swirl numbers $0.5,0.75$ and 1.0 
Figure 22. Comparisons of temperature pdfs at $x=155 \mathrm{~mm}$ at equidistant radial locations $\mathrm{r}=0 \mathrm{~mm}, 12 \mathrm{~mm}, 24 \mathrm{~mm}$ and $32 \mathrm{~mm}$. Here solid line, dashed line and inverted triangles denote results for swirl numbers $0.5,0.75$ and 1.0

Figure 23. Radial profiles of temperature intermittency at $x=30 \mathrm{~mm}, 55 \mathrm{~mm}, 100 \mathrm{~mm}$ and $155 \mathrm{~mm}$. Here circles, squares and inverted triangles denote results for swirl numbers 0.5 , 0.75 and 1.0 
Figures

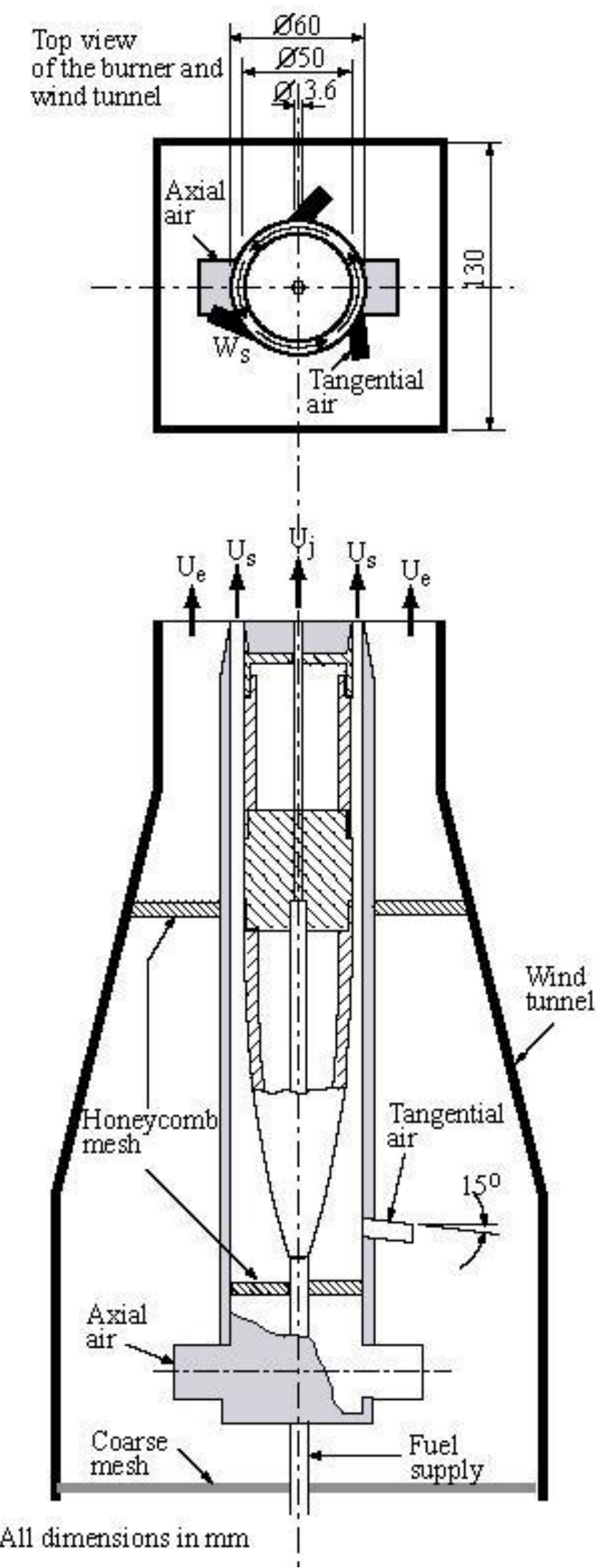

Figure 1. Schematic drawing of the Sydney swirl burner 


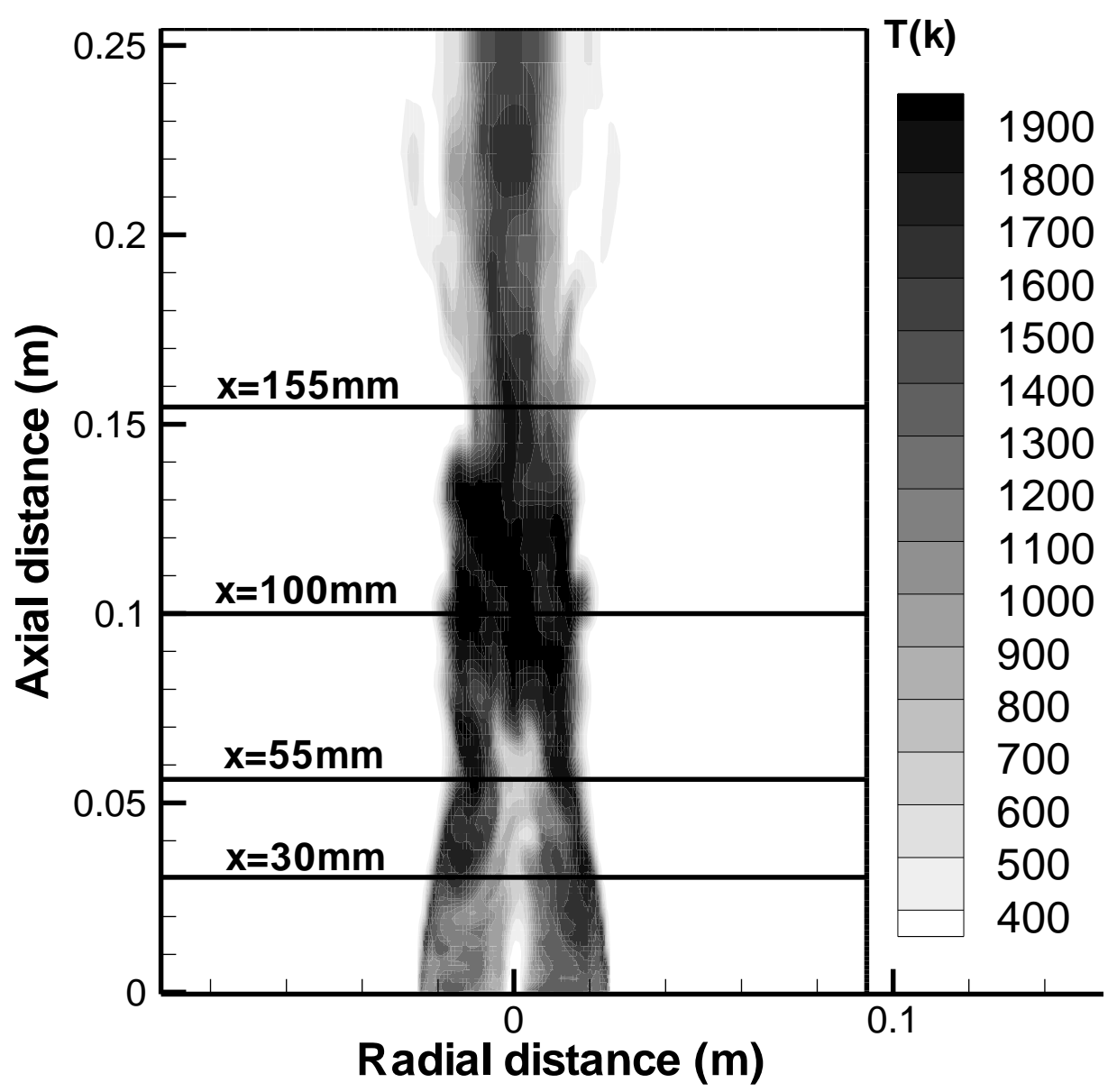

Figure 2. Snapshot of SM1 flame temperature 


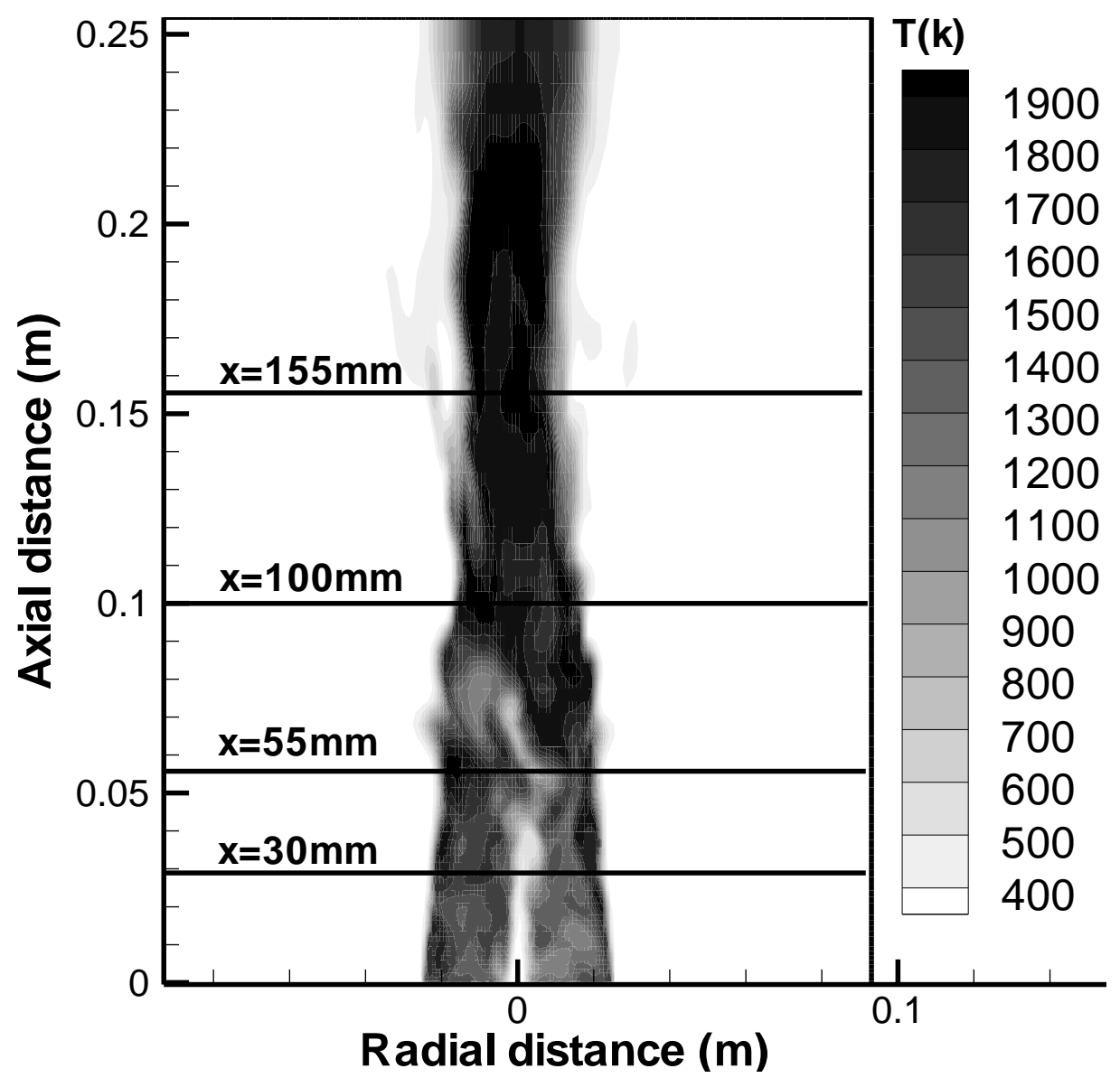

Figure 3. Snapshot of SM1a flame temperature 


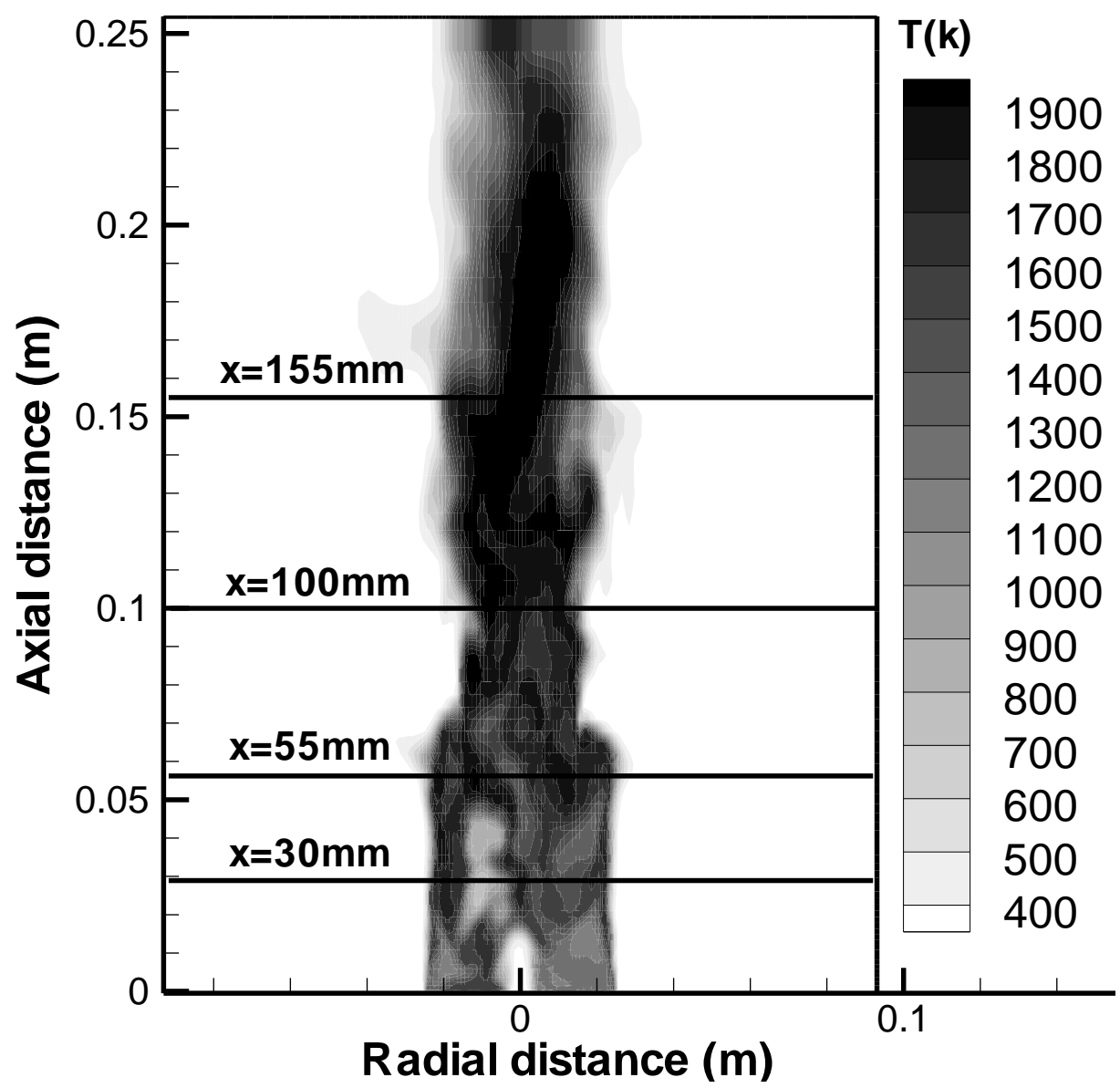

Figure 4. Snapshot of flame SM1b flame temperature 

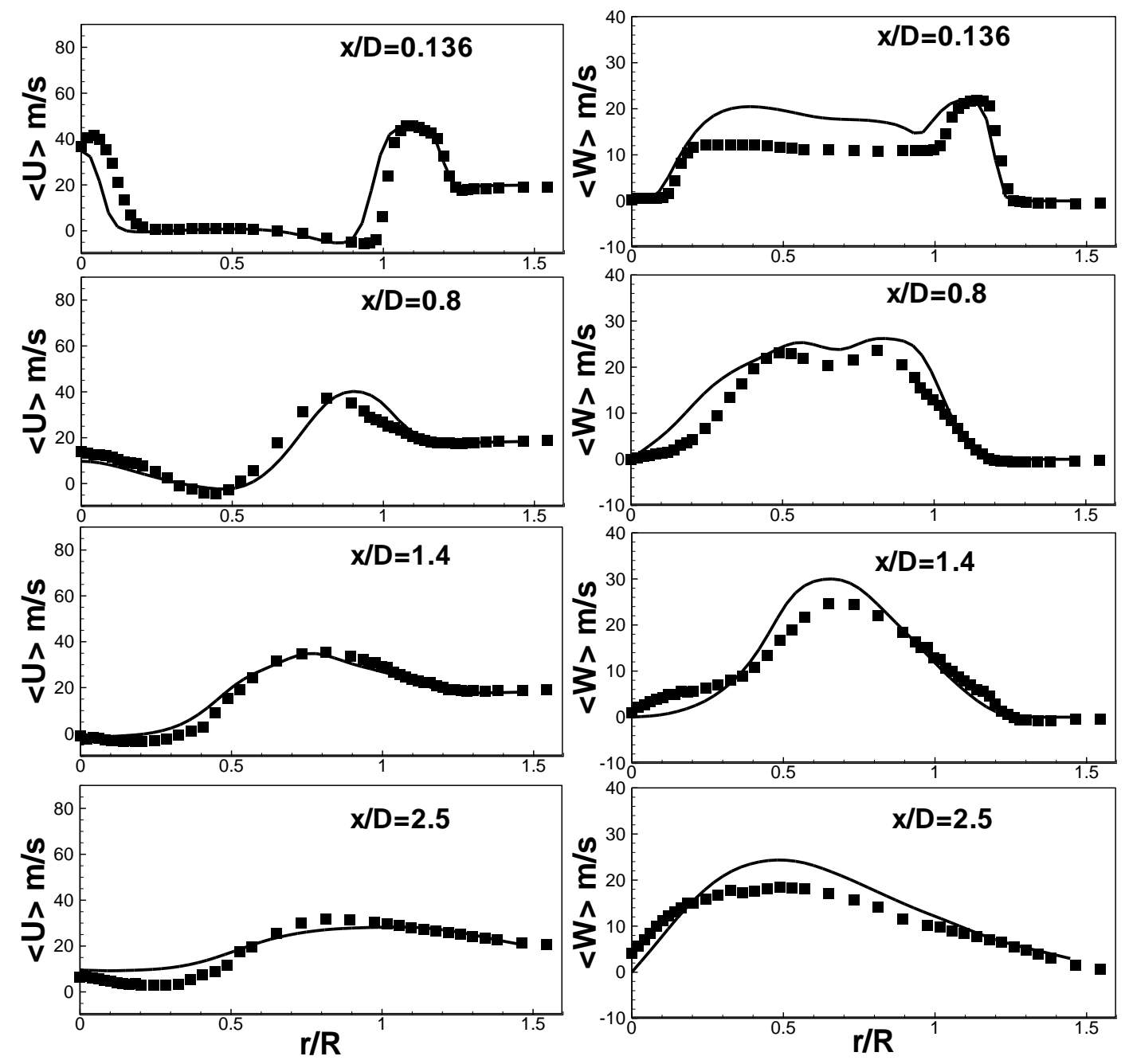

Figure 5. Comparison of mean axial velocity $\langle\mathrm{U}\rangle \mathrm{m} / \mathrm{s}$ and mean swirling velocity $\langle\mathrm{W}\rangle \mathrm{m} / \mathrm{s}$, Lines represent LES results, and symbols represent experimental measurements. 


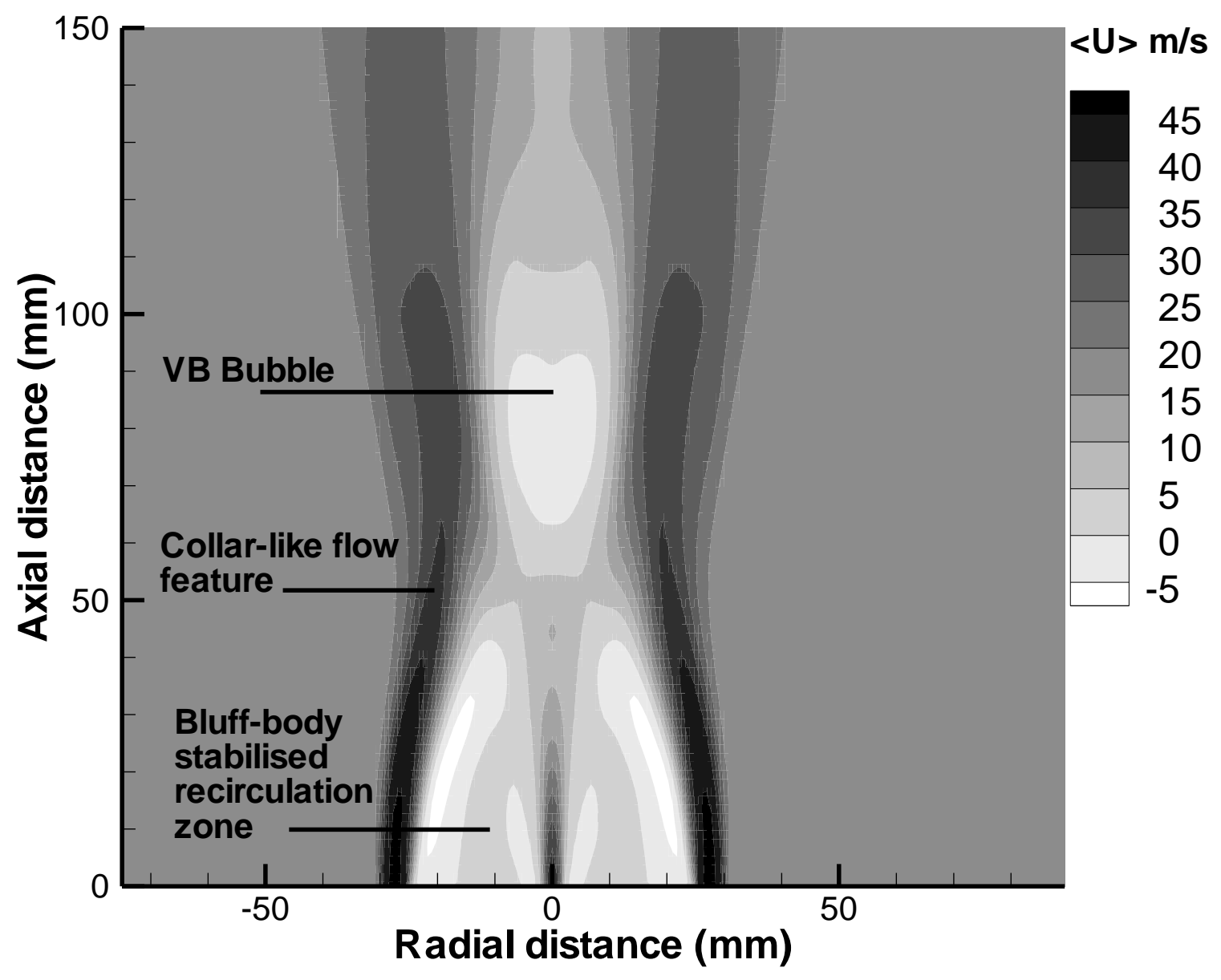

Figure 6. Contour plot of SM1 time-averaged mean axial velocity 

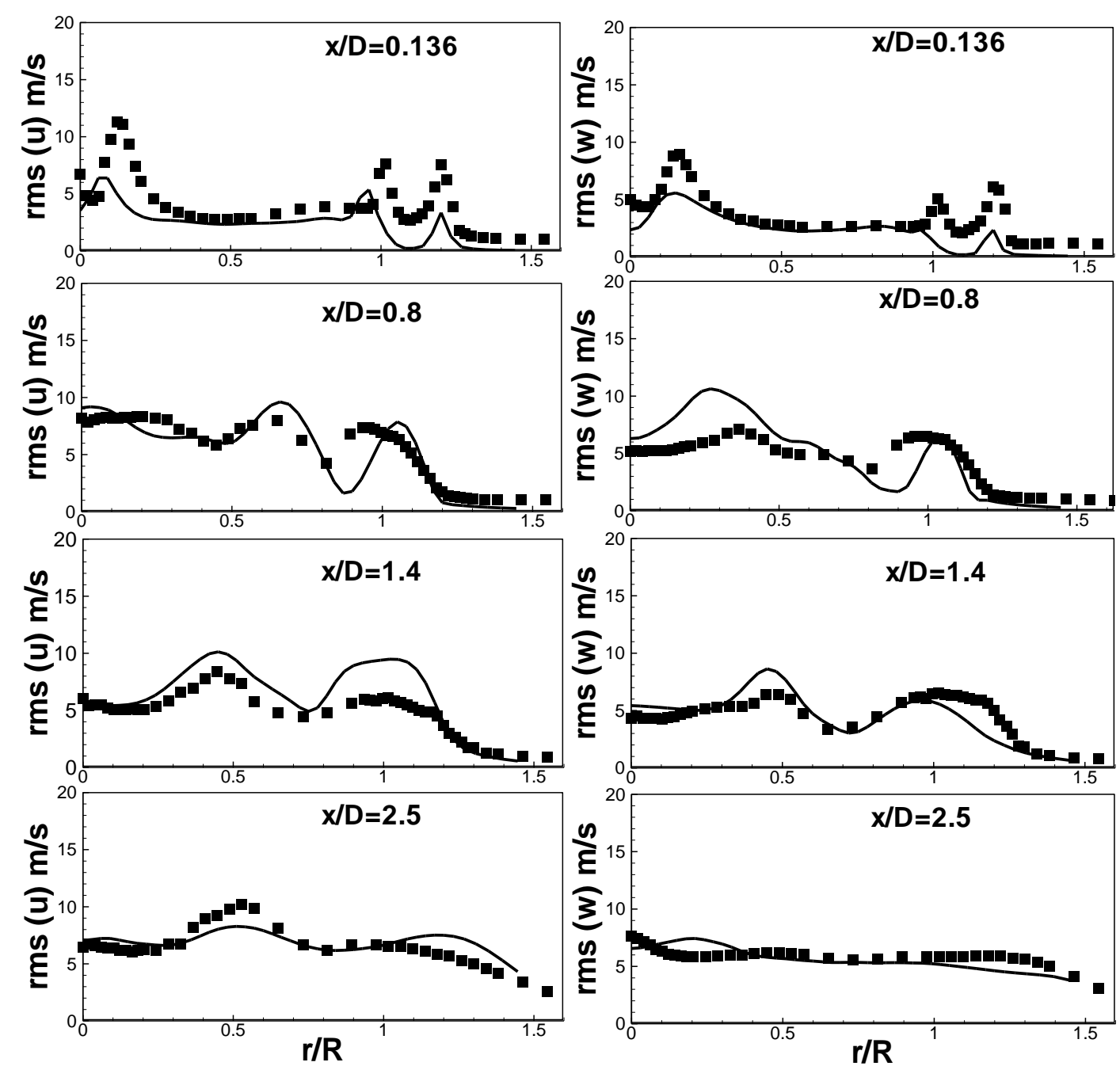

Figure 7. Comparison of rms axial velocity $\mathrm{rms}$ (u) $\mathrm{m} / \mathrm{s}$ and $\mathrm{rms}$ swirling velocity $\mathrm{rms}(\mathrm{w})$ $\mathrm{m} / \mathrm{s}$. Lines represent LES results, and symbols represent experimental measurements. 

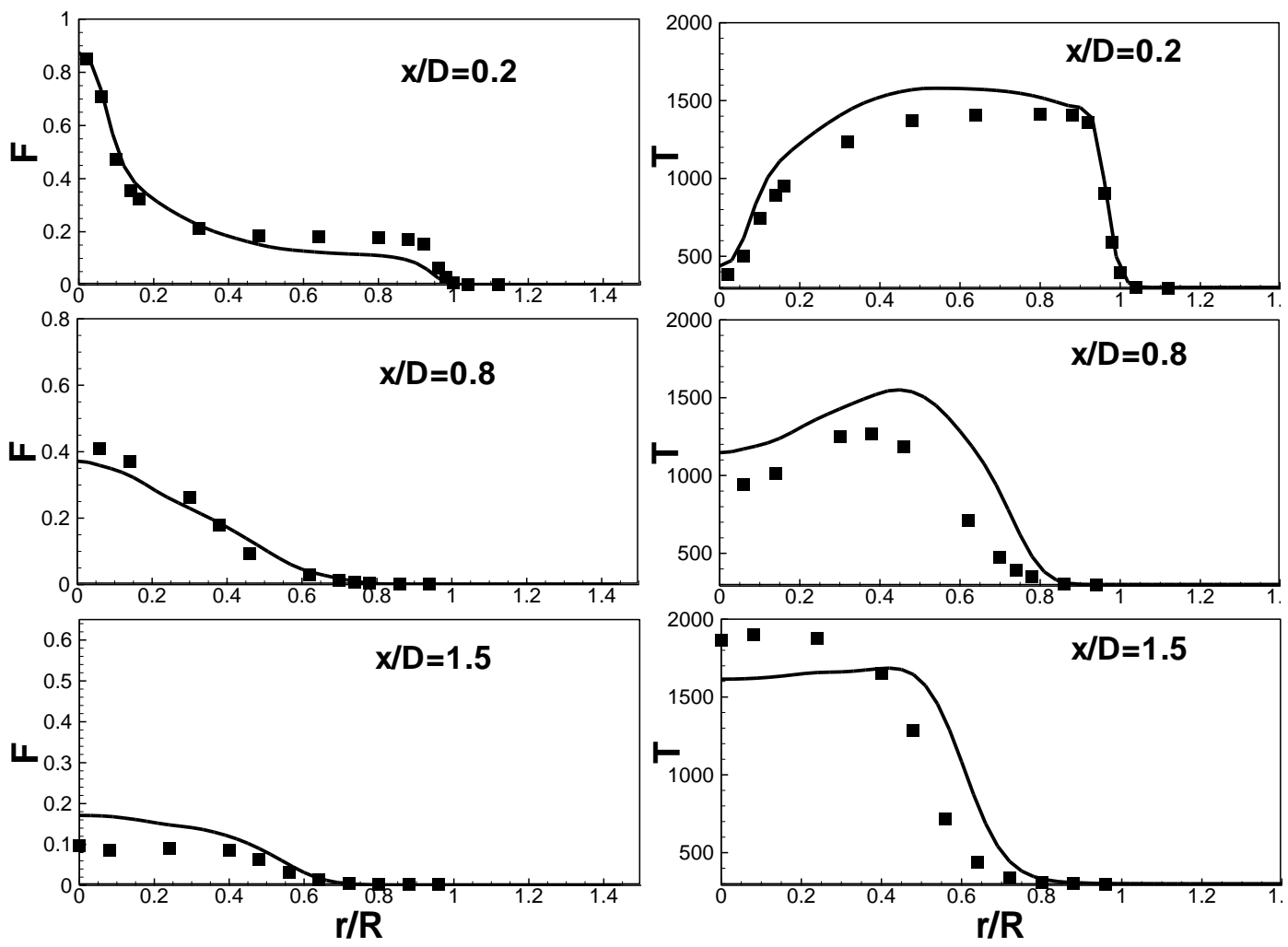

Figure 8. Comparison of mean mixture fraction $F$ and mean temperature $T(K)$. Lines represent LES results, and symbols represent experimental measurements. 

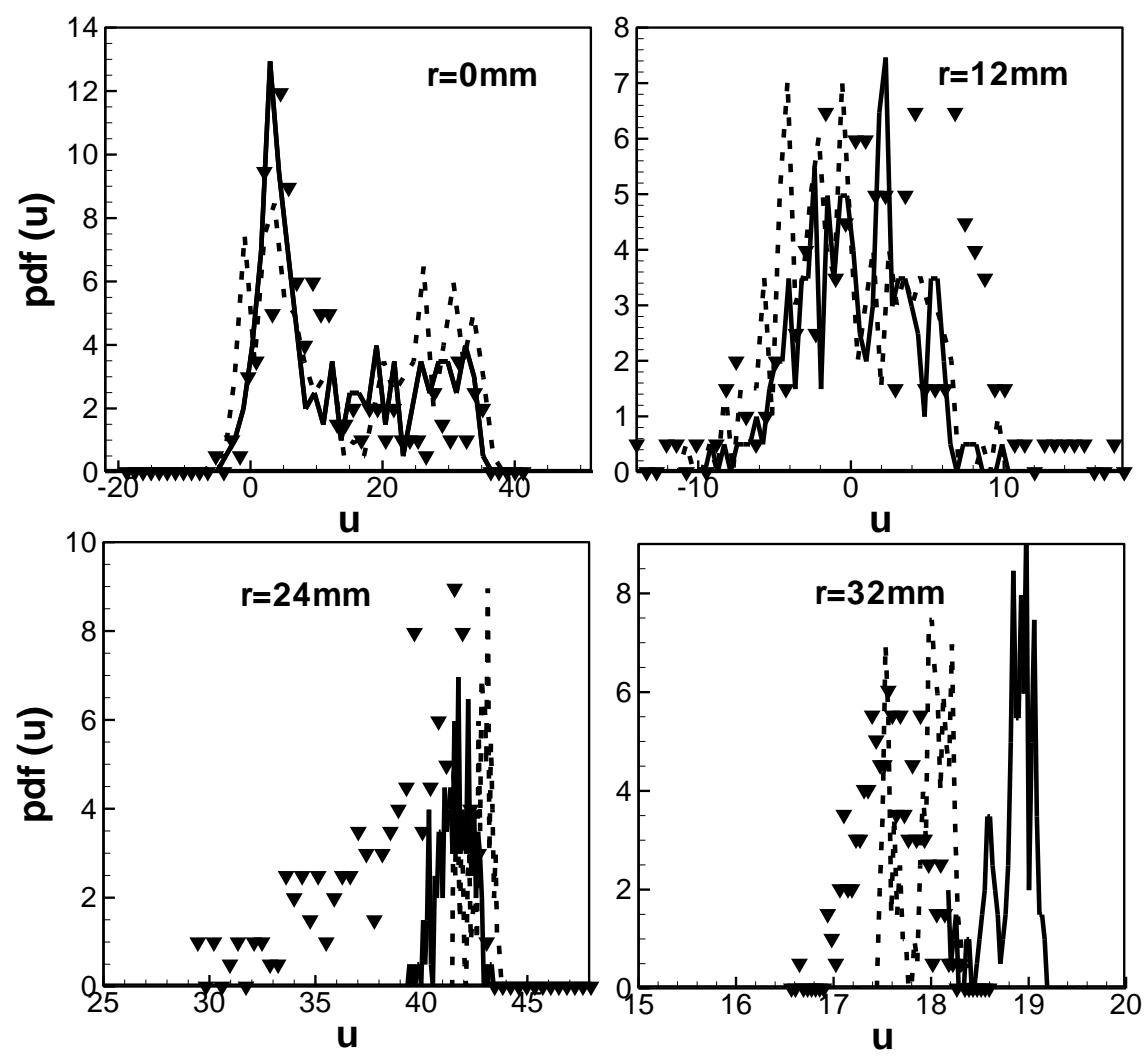

Figure 9. Comparisons of velocity pdfs at $\mathrm{x}=30 \mathrm{~mm}$ at equidistant radial locations $\mathrm{r}=0 \mathrm{~mm}$, $12 \mathrm{~mm}, 24 \mathrm{~mm}$ and $32 \mathrm{~mm}$. Here solid line, dashed line and inverted triangles denote results for swirl numbers $0.5,0.75$ and 1.0 

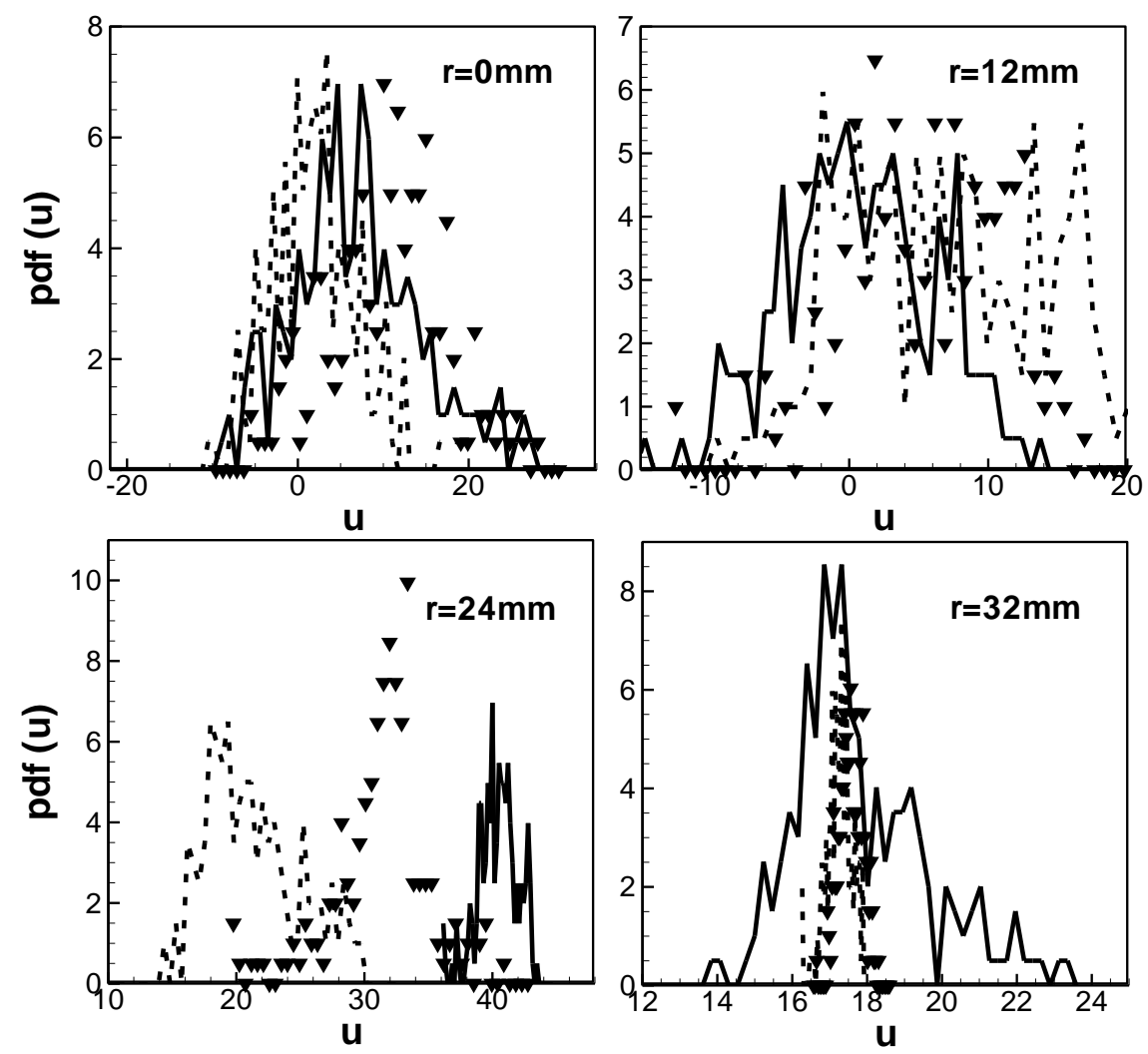

Figure 10. Comparisons of velocity pdfs at $\mathrm{x}=55 \mathrm{~mm}$ at equidistant radial locations $\mathrm{r}=0 \mathrm{~mm}$, $12 \mathrm{~mm}, 24 \mathrm{~mm}$ and $32 \mathrm{~mm}$. Here solid line, dashed line and inverted triangles denote results for swirl numbers $0.5,0.75$ and 1.0 

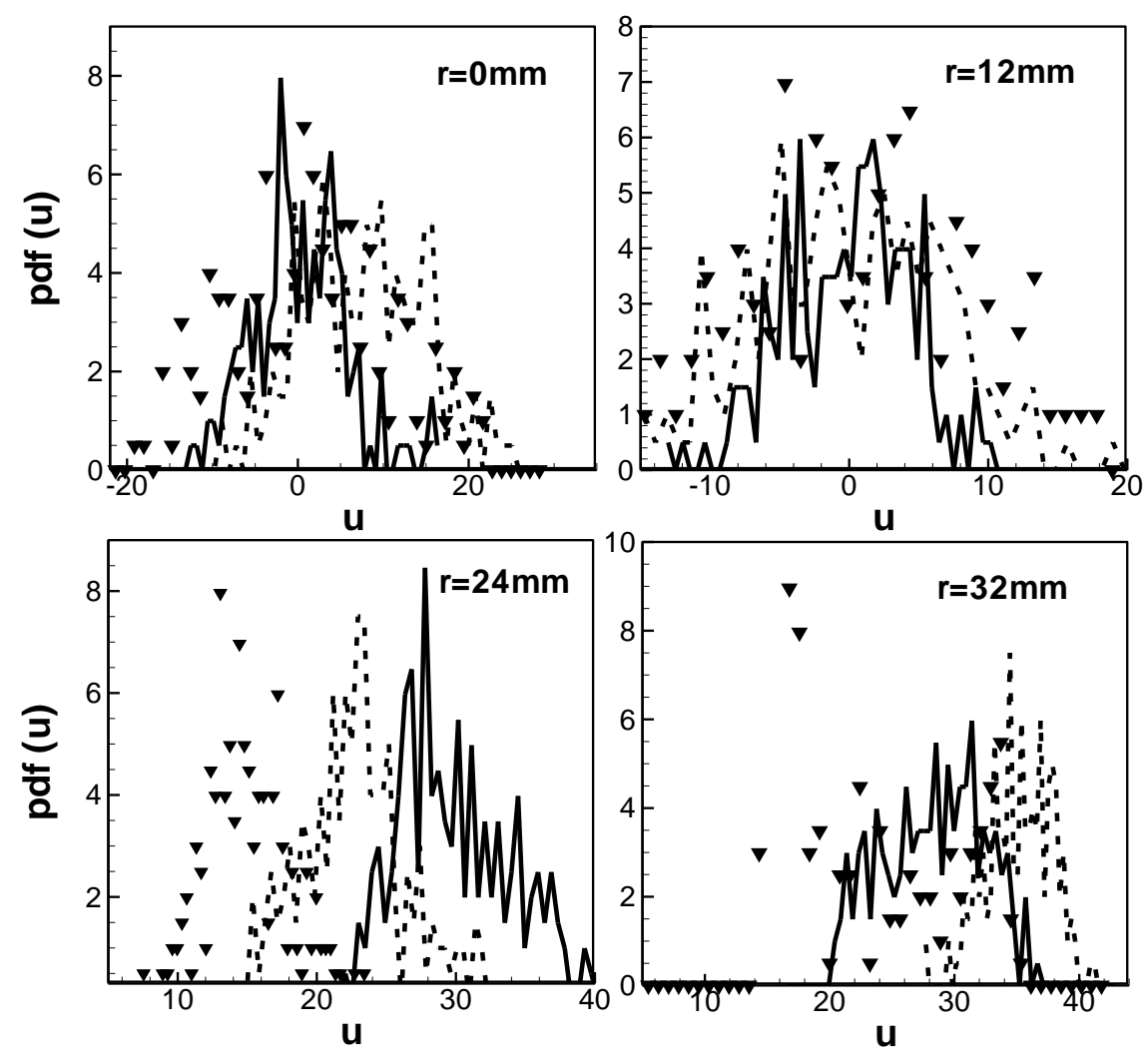

Figure 11. Comparisons of velocity pdfs at $\mathrm{x}=100 \mathrm{~mm}$ at equidistant radial locations $\mathrm{r}=0 \mathrm{~mm}$, $12 \mathrm{~mm}, 24 \mathrm{~mm}$ and $32 \mathrm{~mm}$. Here solid line, dashed line and inverted triangles denote results for swirl numbers $0.5,0.75$ and 1.0 

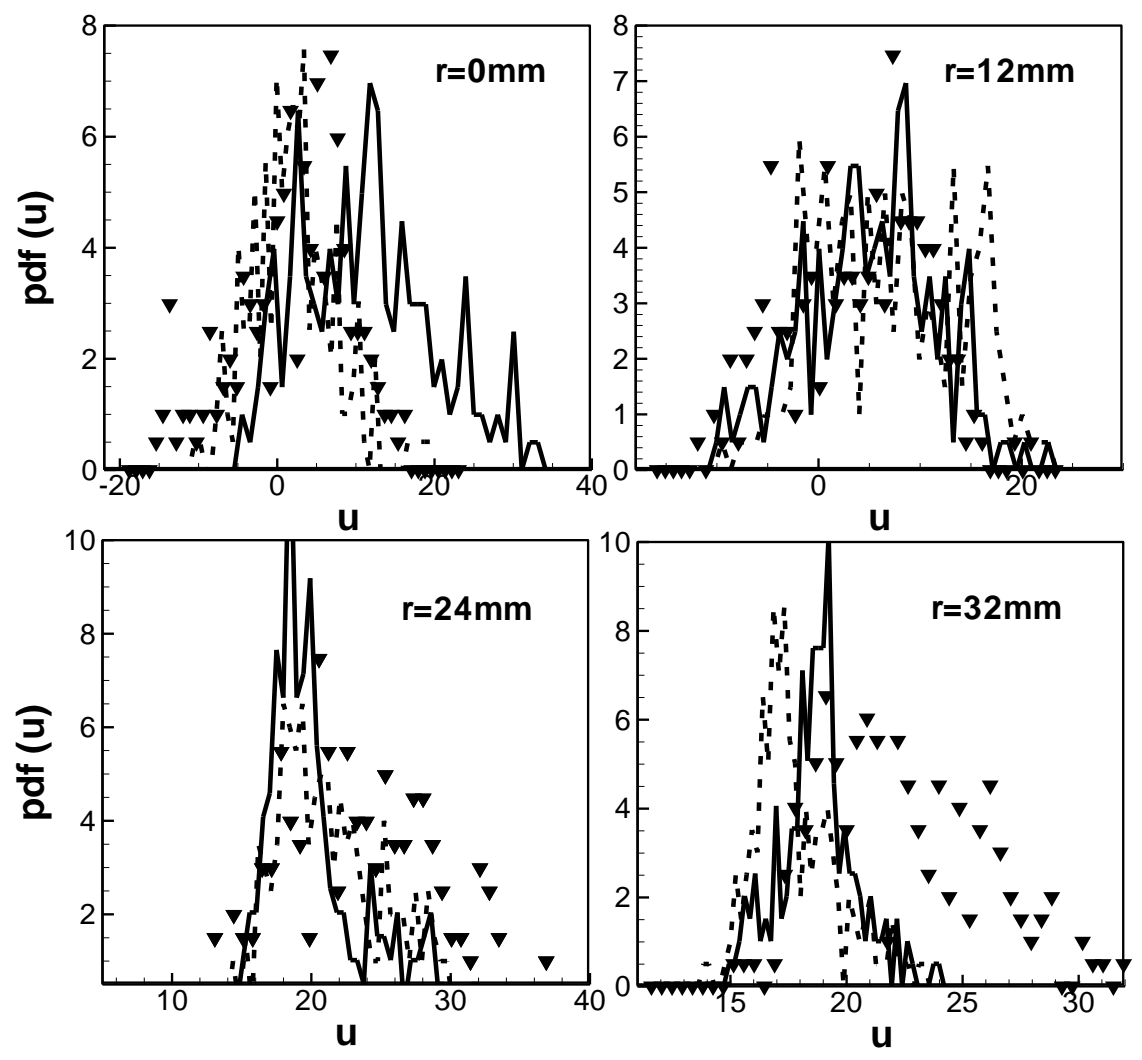

Figure 12. Comparisons of velocity pdfs at $x=155 \mathrm{~mm}$ at equidistant radial locations $\mathrm{r}=0 \mathrm{~mm}$, $12 \mathrm{~mm}, 24 \mathrm{~mm}$ and $32 \mathrm{~mm}$. Here solid line, dashed line and inverted triangles denote results for swirl numbers $0.5,0.75$ and 1.0 

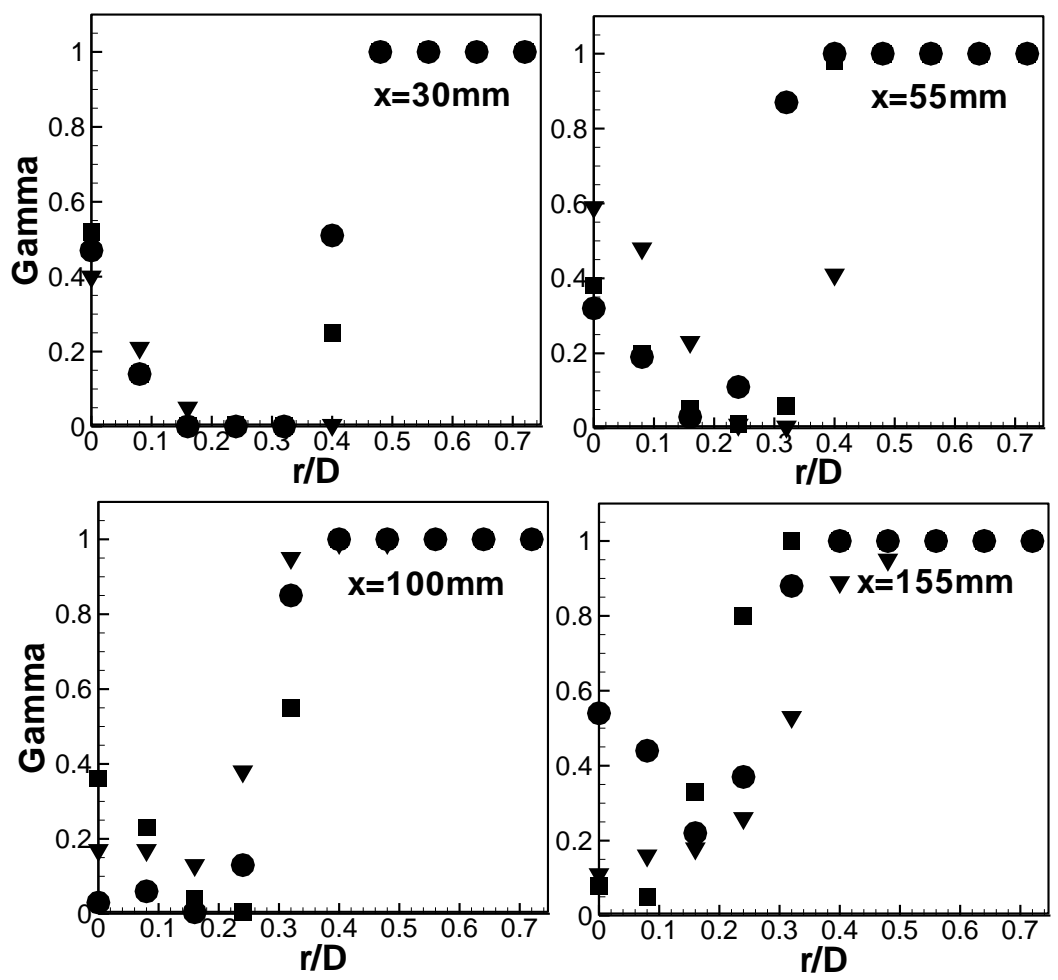

Figure 13. Radial profiles of velocity intermittency at $x=30 \mathrm{~mm}, 55 \mathrm{~mm}, 100 \mathrm{~mm}$ and $155 \mathrm{~mm}$. Here circles, squares and inverted triangles denote results for swirl numbers $0.5,0.75$ and 1.0 

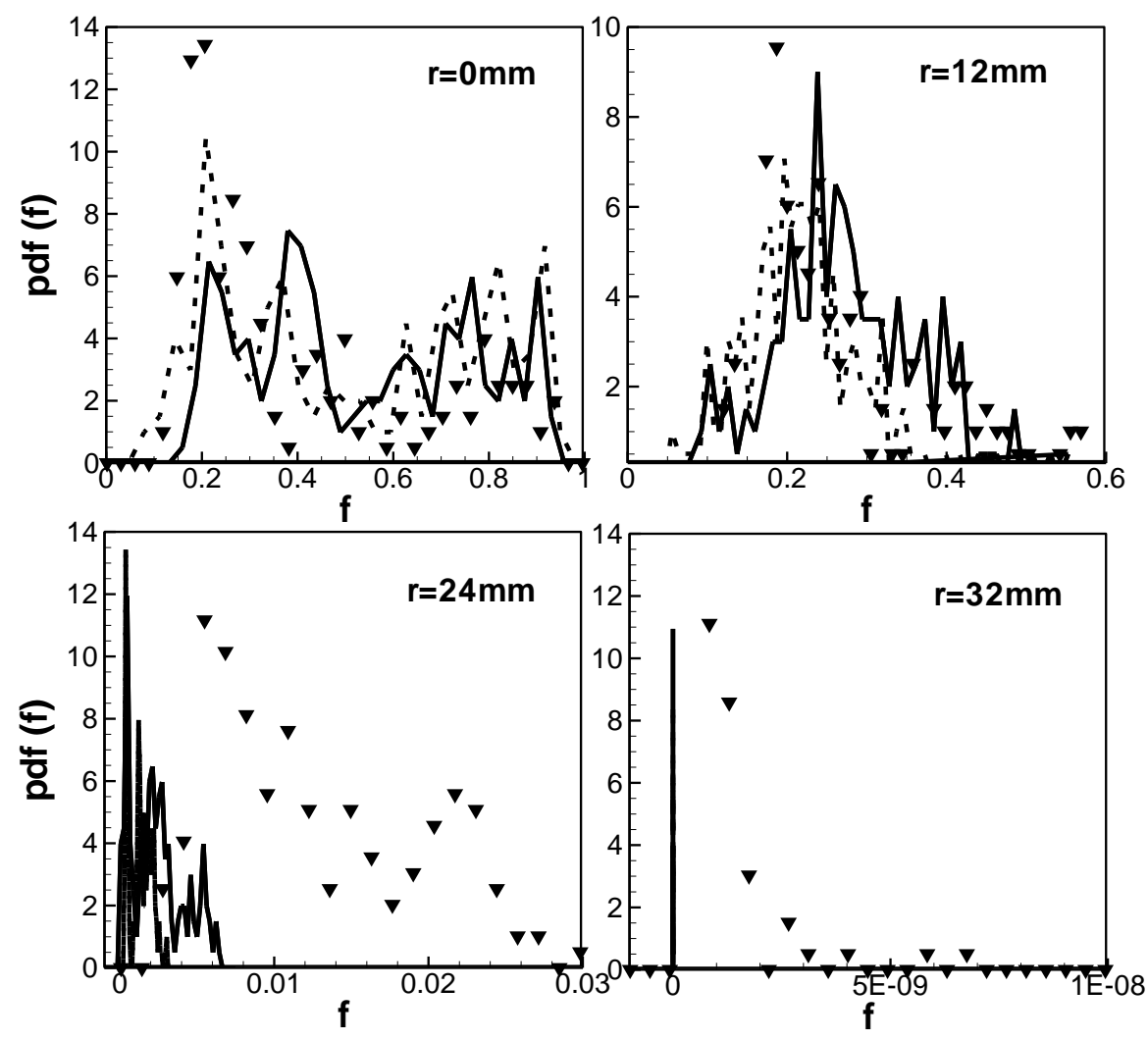

Figure 14. Comparisons of mixture fraction pdfs at $\mathrm{x}=30 \mathrm{~mm}$ at equidistant radial locations $\mathrm{r}=0 \mathrm{~mm}, 12 \mathrm{~mm}, 24 \mathrm{~mm}$ and $32 \mathrm{~mm}$. Here solid line, dashed line and inverted triangles denote results for swirl numbers $0.5,0.75$ and 1.0 

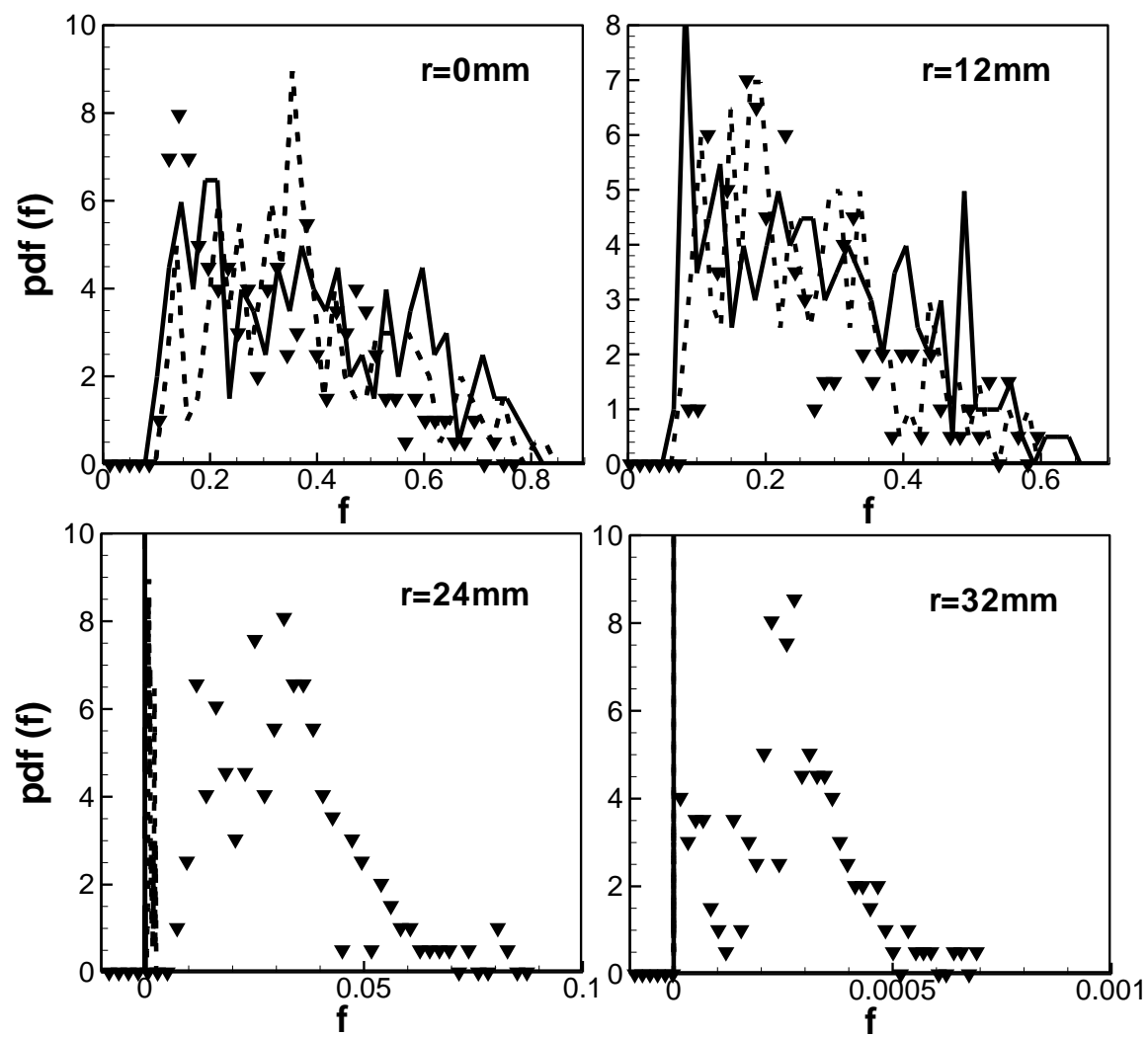

Figure 15. Comparisons of mixture fraction pdfs at $\mathrm{x}=55 \mathrm{~mm}$ at equidistant radial locations $\mathrm{r}=0 \mathrm{~mm}, 12 \mathrm{~mm}, 24 \mathrm{~mm}$ and $32 \mathrm{~mm}$. Here solid line, dashed line and inverted triangles denote results for swirl numbers $0.5,0.75$ and 1.0 

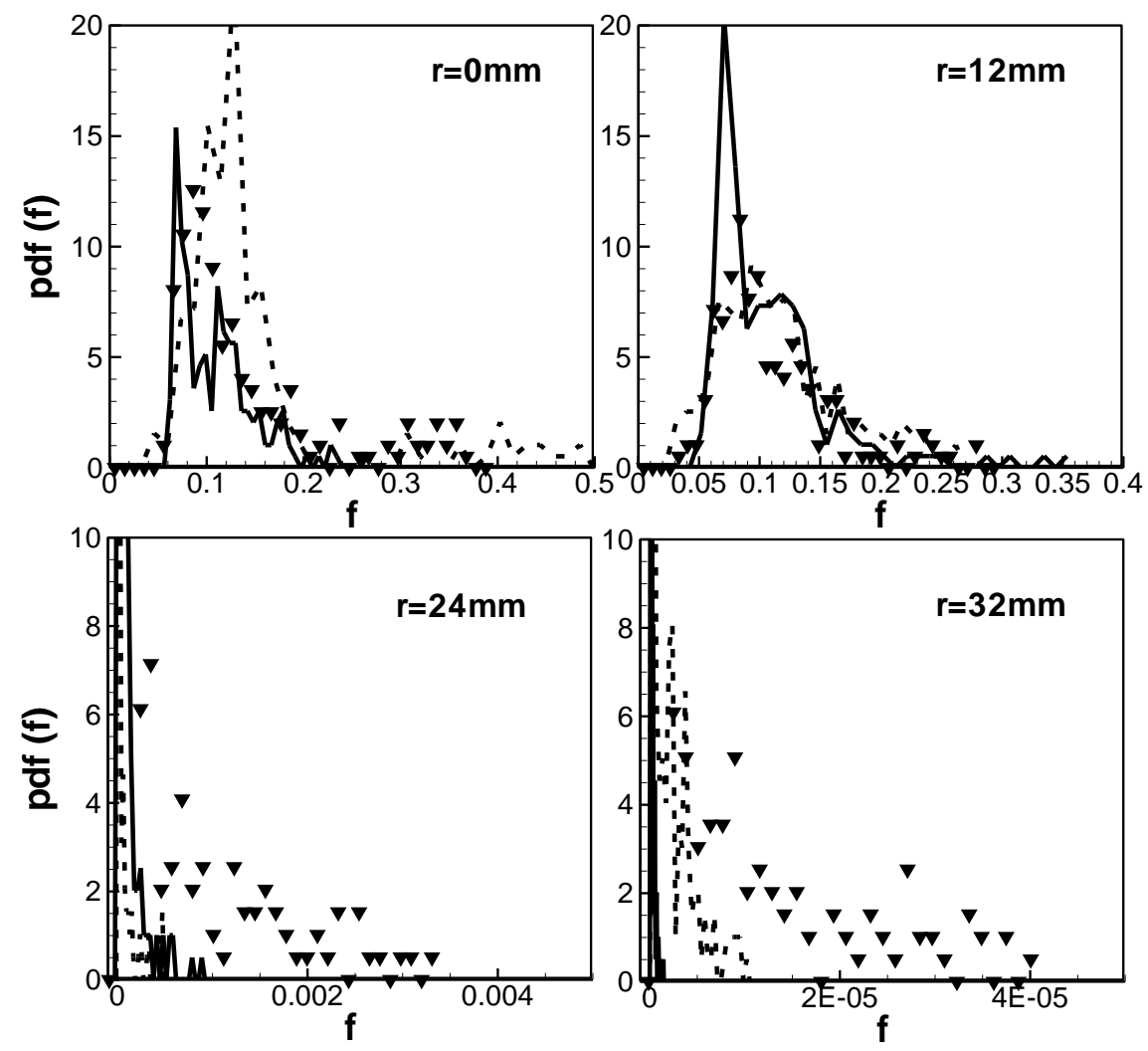

Figure 16. Comparisons of mixture fraction pdfs at $\mathrm{x}=100 \mathrm{~mm}$ at equidistant radial locations $\mathrm{r}=0 \mathrm{~mm}, 12 \mathrm{~mm}, 24 \mathrm{~mm}$ and $32 \mathrm{~mm}$. Here solid line, dashed line and inverted triangles denote results for swirl numbers $0.5,0.75$ and 1.0 

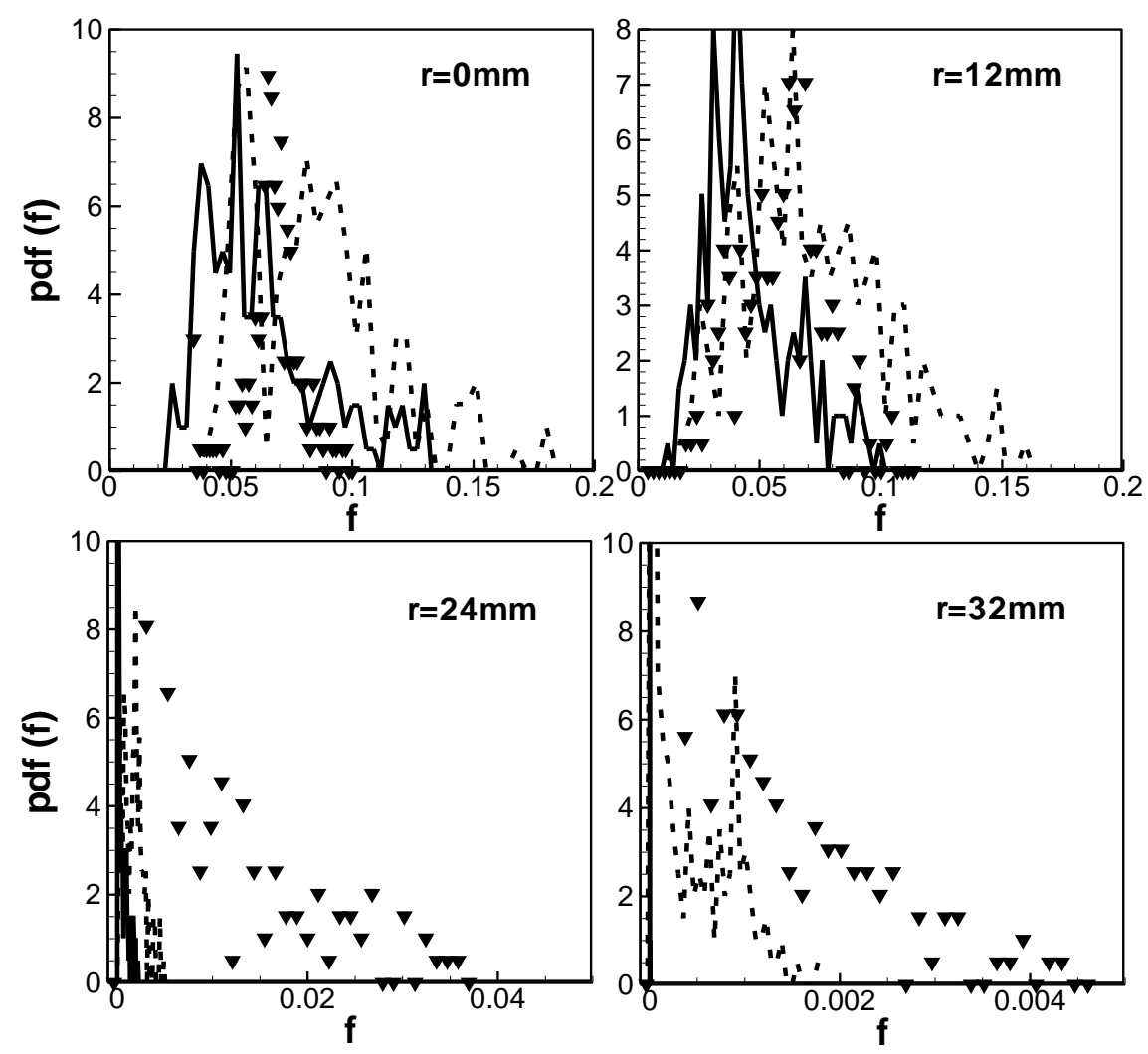

Figure 17. Comparisons of mixture fraction pdfs at $\mathrm{x}=155 \mathrm{~mm}$ at equidistant radial locations $\mathrm{r}=0 \mathrm{~mm}, 12 \mathrm{~mm}, 24 \mathrm{~mm}$ and $32 \mathrm{~mm}$. Here solid line, dashed line and inverted triangles denote results for swirl numbers $0.5,0.75$ and 1.0 

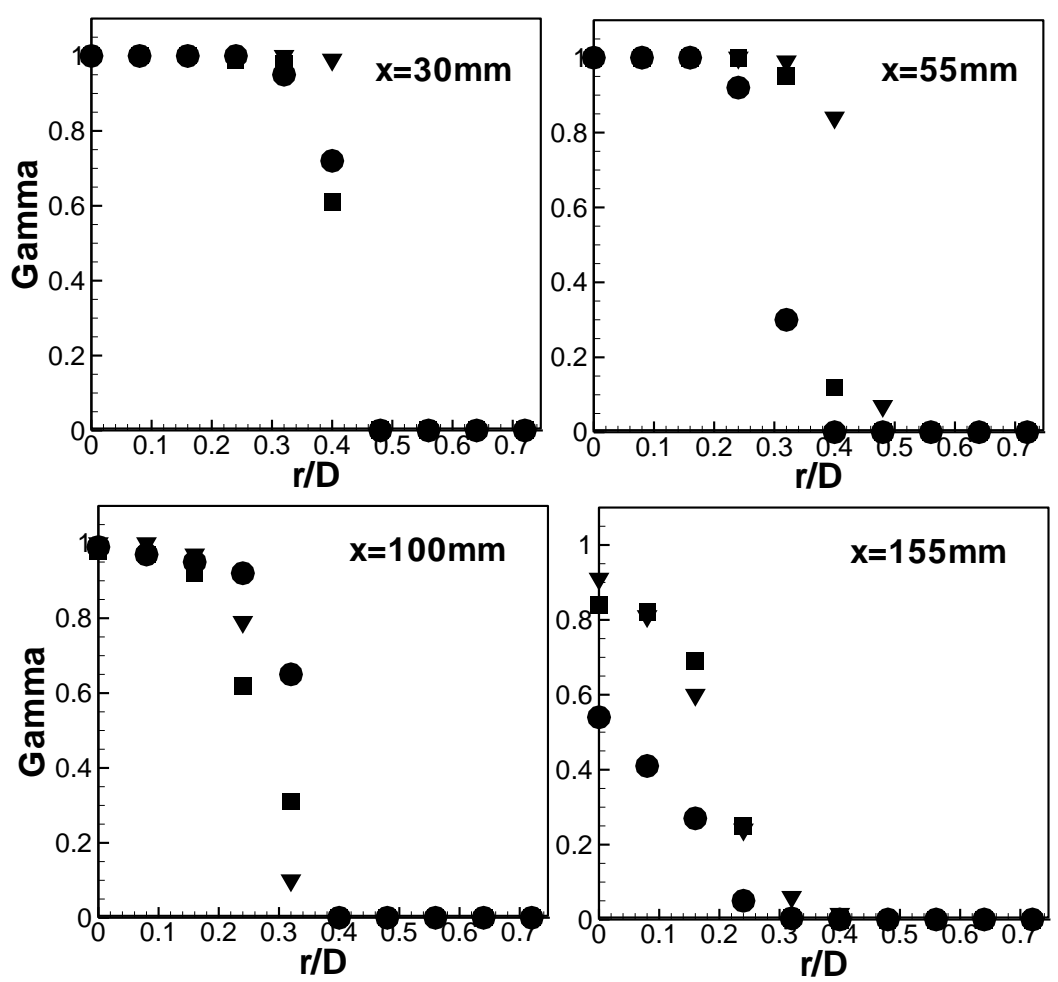

Figure 18. Radial profiles of mixture fraction intermittency at $x=30 \mathrm{~mm}, 55 \mathrm{~mm}, 100 \mathrm{~mm}$ and $155 \mathrm{~mm}$. Here circles, squares and inverted triangles denote results for swirl numbers 0.5 , 0.75 and 1.0 

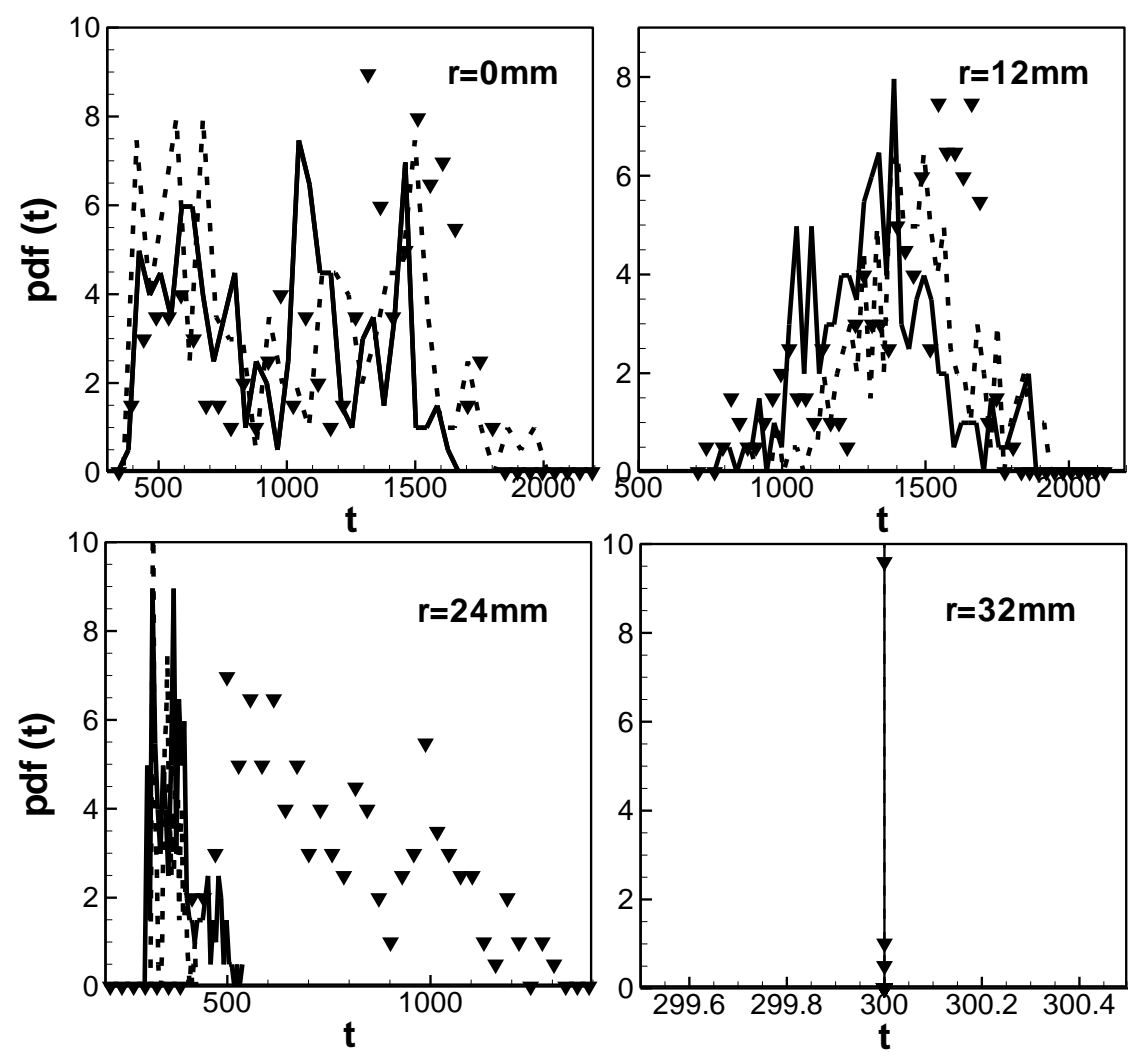

Figure 19. Comparisons of temperature pdfs at $x=30 \mathrm{~mm}$ at equidistant radial locations $\mathrm{r}=0 \mathrm{~mm}, 12 \mathrm{~mm}, 24 \mathrm{~mm}$ and $32 \mathrm{~mm}$. Here solid line, dashed line and inverted triangles denote results for swirl numbers $0.5,0.75$ and 1.0 

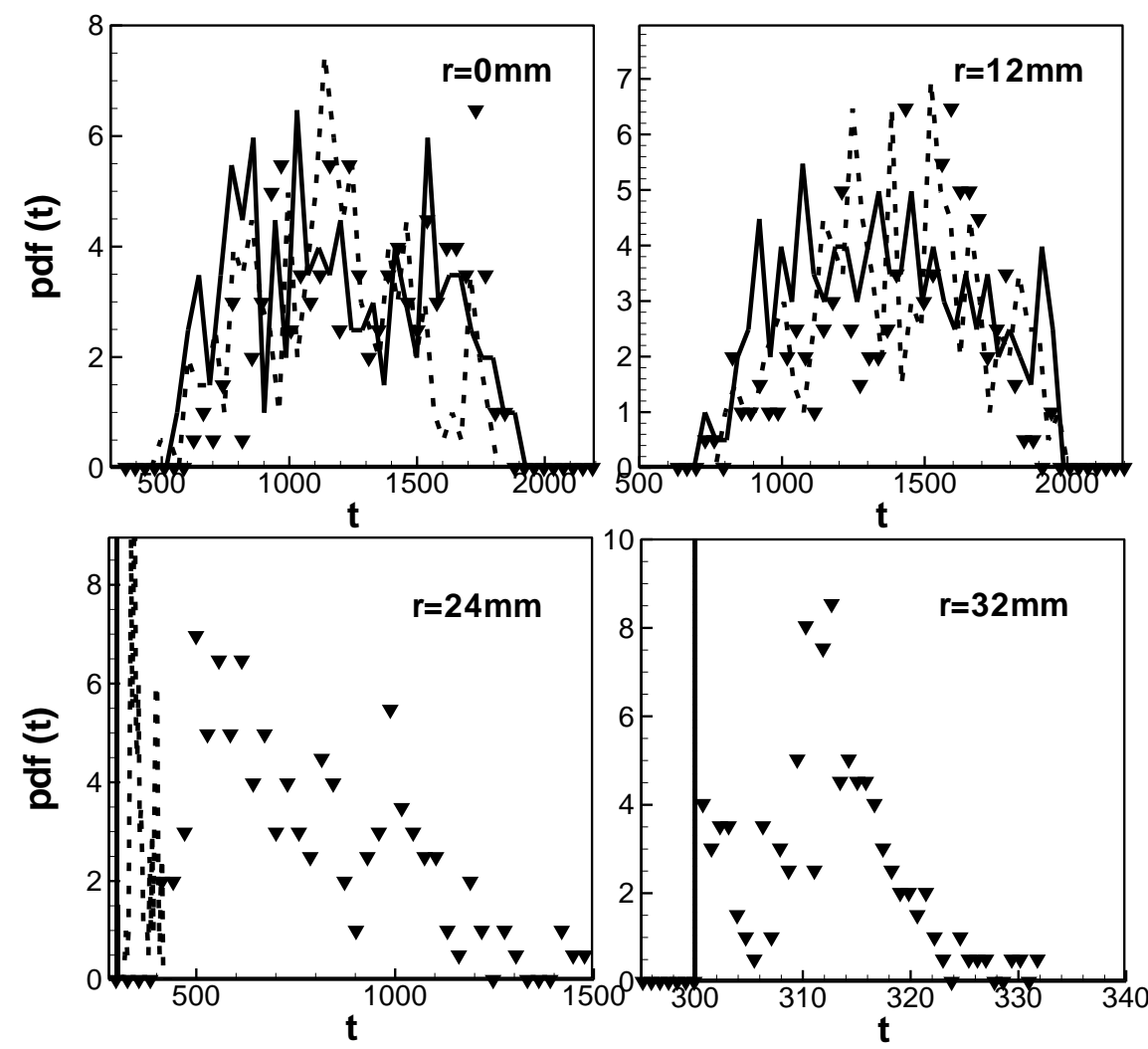

Figure 20. Comparisons of temperature pdfs at $\mathrm{x}=55 \mathrm{~mm}$ at equidistant radial locations $\mathrm{r}=0 \mathrm{~mm}, 12 \mathrm{~mm}, 24 \mathrm{~mm}$ and $32 \mathrm{~mm}$. Here solid line, dashed line and inverted triangles denote results for swirl numbers $0.5,0.75$ and 1.0 

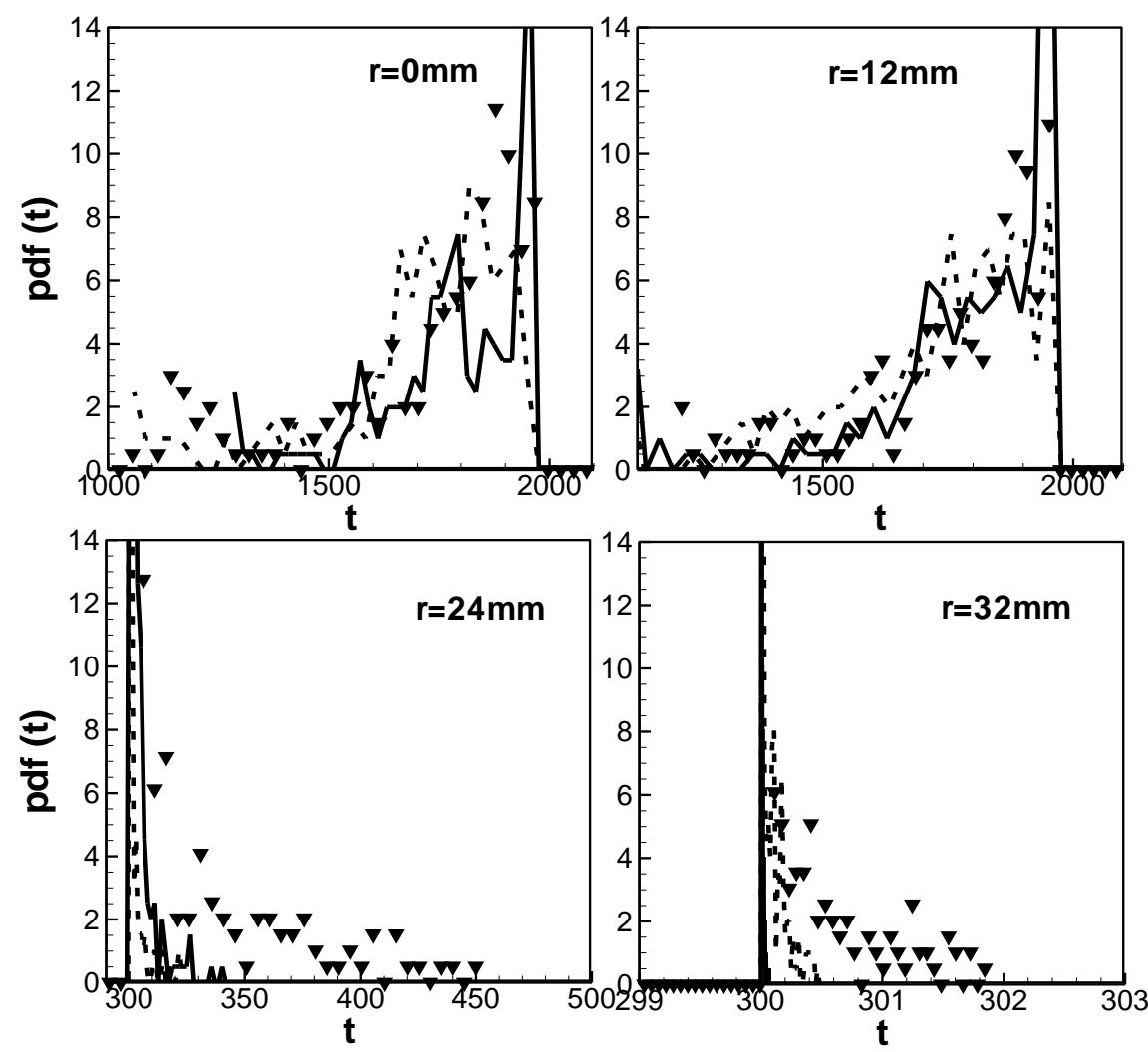

Figure 21. Comparisons of temperature pdfs at $x=100 \mathrm{~mm}$ at equidistant radial locations $\mathrm{r}=0 \mathrm{~mm}, 12 \mathrm{~mm}, 24 \mathrm{~mm}$ and $32 \mathrm{~mm}$. Here solid line, dashed line and inverted triangles denote results for swirl numbers $0.5,0.75$ and 1.0 

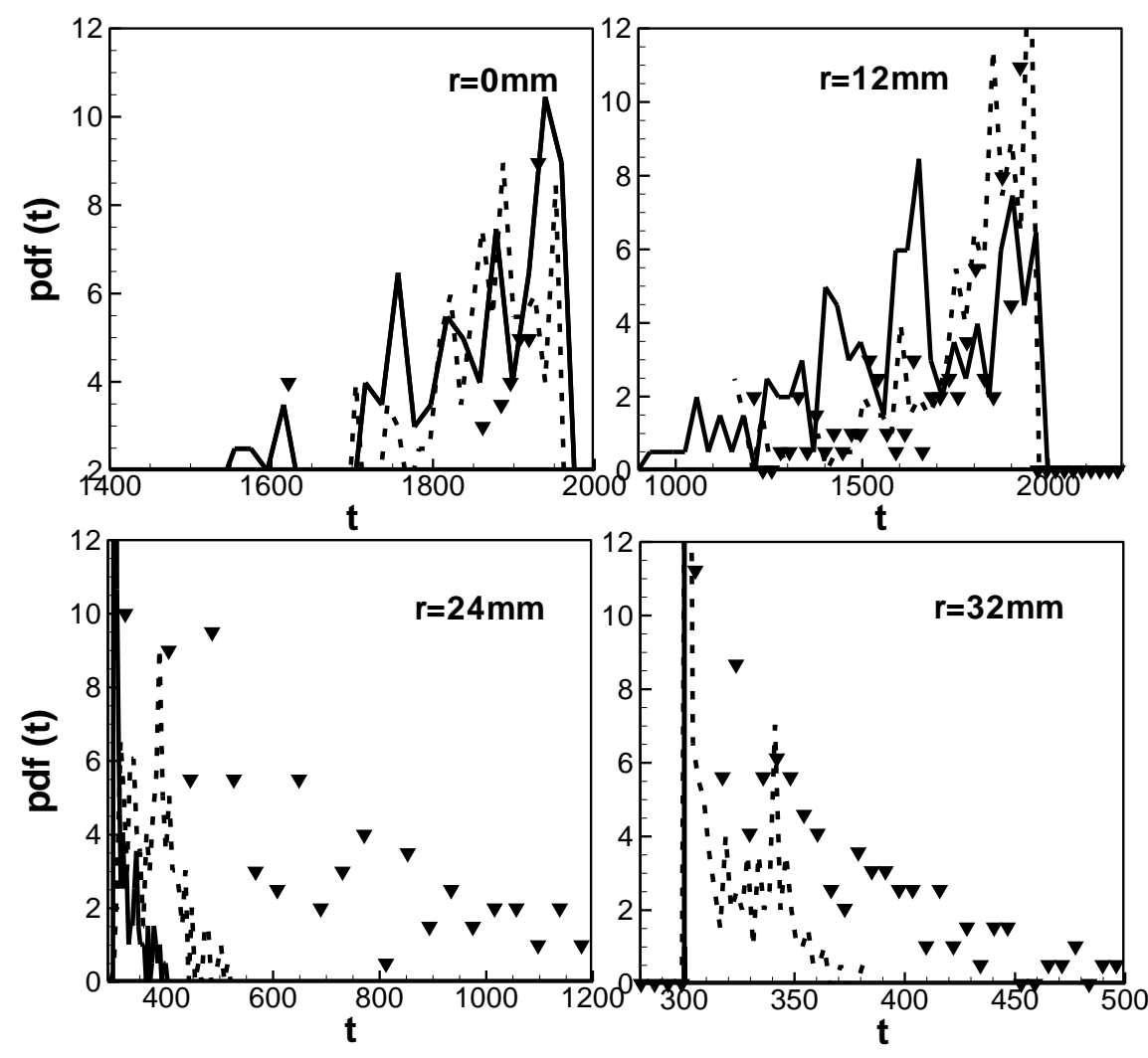

Figure 22. Comparisons of temperature pdfs at $x=155 \mathrm{~mm}$ at equidistant radial locations $\mathrm{r}=0 \mathrm{~mm}, 12 \mathrm{~mm}, 24 \mathrm{~mm}$ and $32 \mathrm{~mm}$. Here solid line, dashed line and inverted triangles denote results for swirl numbers $0.5,0.75$ and 1.0 

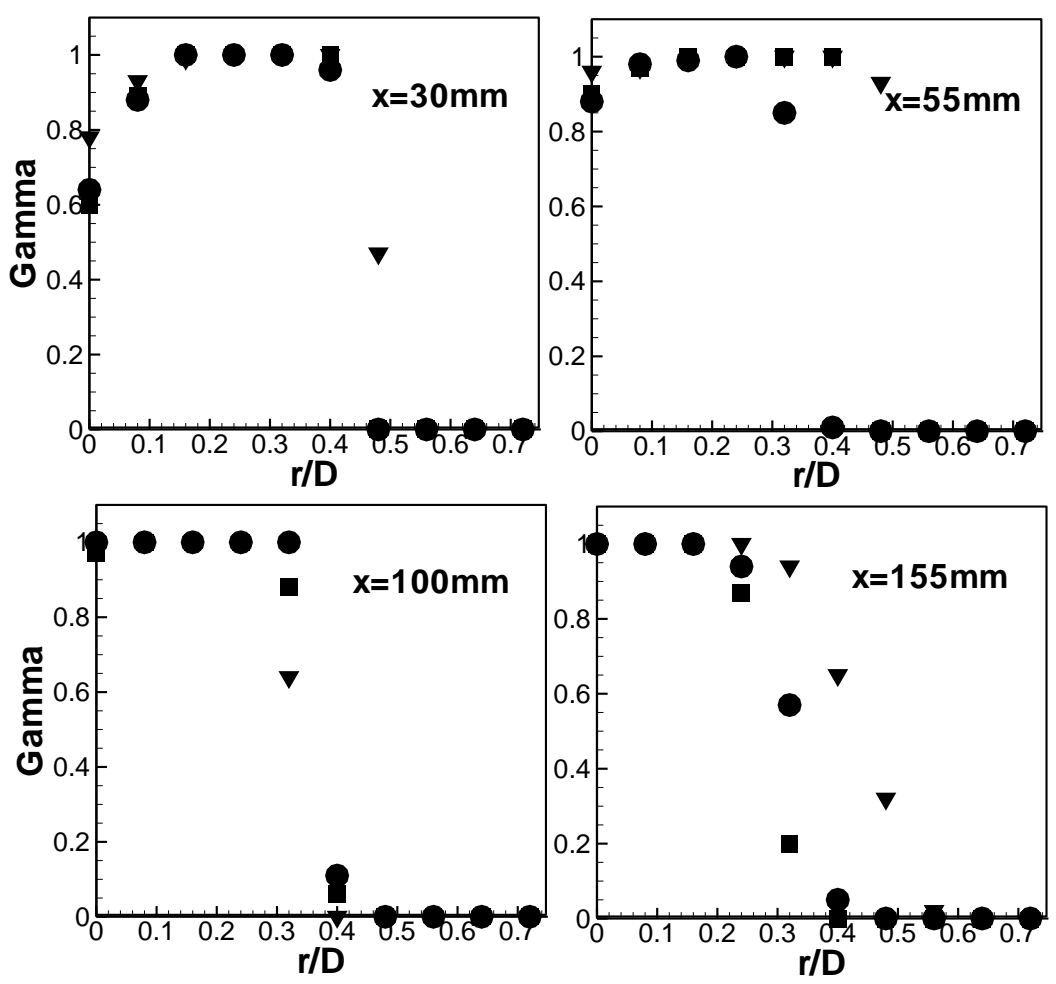

Figure 23. Radial profiles of temperature intermittency at $x=30 \mathrm{~mm}, 55 \mathrm{~mm}, 100 \mathrm{~mm}$ and $155 \mathrm{~mm}$. Here circles, squares and inverted triangles denote results for swirl numbers 0.5 , 0.75 and 1.0 